\title{
SEARCHING FOR DARK MATTER ANNIHILATION IN RECENTLY DISCOVERED MILKY WAY SATELLITES
} WITH FERMI-LAT

A. Albert ${ }^{1}$, B. Anderson ${ }^{2,3}$, K. Bechtol ${ }^{4}$, A. Drlica-Wagner ${ }^{5}$, M. Meyer ${ }^{2,3}$, M. Sánchez-Conde ${ }^{2,3}$, L. Strigari ${ }^{6}$,

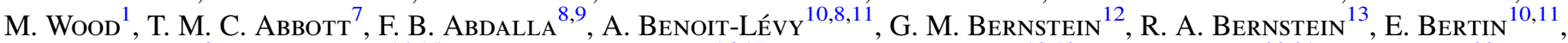
D. Brooks $^{8}$, D. L. Burke ${ }^{14,15}$, A. Carnero Rosell ${ }^{16,17}$, M. Carrasco Kind ${ }^{18,19}$, J. CArretero ${ }^{20,21}$, M. Crocce ${ }^{20}$, C. E. Cunha ${ }^{14}$, C. B. D’Andrea ${ }^{22,23}$, L. N. Da Costa ${ }^{16,17}$, S. Desai ${ }^{24,25}$, H. T. Diehl ${ }^{5}$, J. P. Dietrich ${ }^{24,25}$, P. Doel ${ }^{8}$, T. F. Eifler ${ }^{12,26}$, A. E. Evrard ${ }^{27,28}$, A. Fausti Neto ${ }^{16}$, D. A. Finley ${ }^{5}$, B. Flaugher ${ }^{5}$, P. Fosalba ${ }^{20}$, J. Frieman ${ }^{5,29}$,

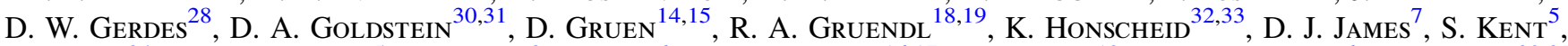
K. KuehN ${ }^{34}$, N. Kuropatkin ${ }^{5}$, O. Lahav ${ }^{8}$, T. S. Li ${ }^{6}$, M. A. G. Maia ${ }^{16,17}$, M. March ${ }^{12}$, J. L. Marshall ${ }^{6}$, P. Martini ${ }^{32,35}$, C. J. Miller ${ }^{27,28}$, R. Miquel ${ }^{21,36}$, E. Neilsen ${ }^{5}$, B. Nord ${ }^{5}$, R. Ogando ${ }^{16,17}$, A. A. Plazas ${ }^{26}$, K. Reil ${ }^{15}$, A. K. Romer ${ }^{37}$, E. S. Rykoff ${ }^{14,15}$, E. Sanchez ${ }^{38}$, B. Santiago ${ }^{16,39}$, M. Schubnell ${ }^{28}$, I. Sevilla-Noarbe ${ }^{18,38}$, R. C. Smith ${ }^{7}$, M. Soares-Santos ${ }^{5}$, F. Sobreira ${ }^{16}$, E. Suchyta ${ }^{12}$, M. E. C. Swanson $^{19}$, G. Tarle $^{28}$, V. Vikram ${ }^{40}$, A. R. Walker ${ }^{7}$, And R. H. WECHSLER ${ }^{14,15,41}$

(The Fermi-LAT and DES Collaborations)

\footnotetext{
${ }^{1}$ Los Alamos National Laboratory, Los Alamos, NM 87545, USA; amalbert@lanl.gov, mdwood@slac.stanford.edu

2 Department of Physics, Stockholm University, AlbaNova, SE-106 91 Stockholm, Sweden; keith.bechtol@icecube.wisc.edu ${ }^{3}$ The Oskar Klein Centre for Cosmoparticle Physics, AlbaNova, SE-106 91 Stockholm, Sweden

${ }^{4}$ Dept. of Physics and Wisconsin IceCube Particle Astrophysics Center, University of Wisconsin, Madison, WI 53706, USA 5 Fermi National Accelerator Laboratory, P.O. Box 500, Batavia, IL 60510, USA; kadrlica@fnal.gov

${ }^{6}$ George P. and Cynthia Woods Mitchell Institute for Fundamental Physics and Astronomy, and Department of Physics and Astronomy, Texas A\&M University, College Station, TX 77843, USA; strigari@physics.tamu.edu

${ }^{7}$ Cerro Tololo Inter-American Observatory, National Optical Astronomy Observatory, Casilla 603, La Serena, Chile

${ }^{8}$ Department of Physics \& Astronomy, University College London, Gower Street, London WC1E 6BT, UK

${ }^{9}$ Department of Physics and Electronics, Rhodes University, P.O. Box 94, Grahamstown, 6140, South Africa

${ }^{10}$ CNRS, UMR 7095, Institut d'Astrophysique de Paris, F-75014, Paris, France

${ }^{11}$ Sorbonne Universités, UPMC Univ Paris 06, UMR 7095, Institut d'Astrophysique de Paris, F-75014, Paris, France

${ }^{12}$ Department of Physics and Astronomy, University of Pennsylvania, Philadelphia, PA 19104, USA

${ }^{13}$ Carnegie Observatories, 813 Santa Barbara Street, Pasadena, CA 91101, USA

${ }^{14}$ Kavli Institute for Particle Astrophysics \& Cosmology, P.O. Box 2450, Stanford University, Stanford, CA 94305, USA ${ }^{15}$ SLAC National Accelerator Laboratory, Menlo Park, CA 94025, USA

${ }^{16}$ Laboratório Interinstitucional de e-Astronomia-LIneA, Rua Gal. José Cristino 77, Rio de Janeiro, RJ-20921-400, Brazil

${ }^{17}$ Observatório Nacional, Rua Gal. José Cristino 77, Rio de Janeiro, RJ-20921-400, Brazil

${ }^{18}$ Department of Astronomy, University of Illinois, 1002 W. Green Street, Urbana, IL 61801, USA

${ }^{19}$ National Center for Supercomputing Applications, 1205 West Clark Street, Urbana, IL 61801, USA

${ }^{20}$ Institut de Ciències de l'Espai, IEEC-CSIC, Campus UAB, Carrer de Can Magrans, s/n, E-08193 Bellaterra, Barcelona, Spain

${ }^{21}$ Institut de Física d'Altes Energies (IFAE), The Barcelona Institute of Science and Technology, Campus UAB, E-08193 Bellaterra (Barcelona) Spain

${ }_{22}^{2}$ Institute of Cosmology \& Gravitation, University of Portsmouth, Portsmouth PO1 3FX, UK

${ }^{23}$ School of Physics and Astronomy, University of Southampton, Southampton SO17 1BJ, UK

${ }^{24}$ Excellence Cluster universe, Boltzmannstr. 2, D-85748 Garching, Germany

${ }^{25}$ Faculty of Physics, Ludwig-Maximilians-Universität, Scheinerstr. 1, D-81679 Munich, Germany

${ }^{26}$ Jet Propulsion Laboratory, California Institute of Technology, 4800 Oak Grove Dr., Pasadena, CA 91109, USA

${ }^{27}$ Department of Astronomy, University of Michigan, Ann Arbor, MI 48109, USA

${ }^{28}$ Department of Physics, University of Michigan, Ann Arbor, MI 48109, USA

${ }^{29}$ Kavli Institute for Cosmological Physics, University of Chicago, Chicago, IL 60637, USA

${ }^{30}$ Department of Astronomy, University of California, Berkeley, 501 Campbell Hall, Berkeley, CA 94720, USA

${ }^{31}$ Lawrence Berkeley National Laboratory, 1 Cyclotron Road, Berkeley, CA 94720, USA

${ }^{32}$ Center for Cosmology and Astro-Particle Physics, The Ohio State University, Columbus, OH 43210, USA

${ }^{33}$ Department of Physics, The Ohio State University, Columbus, OH 43210, USA

${ }^{34}$ Australian Astronomical Observatory, North Ryde, NSW 2113, Australia

${ }^{35}$ Department of Astronomy, The Ohio State University, Columbus, OH 43210, USA

${ }^{36}$ Institució Catalana de Recerca i Estudis Avançats, E-08010 Barcelona, Spain

${ }^{37}$ Department of Physics and Astronomy, Pevensey Building, University of Sussex, Brighton BN1 9QH, UK

${ }^{38}$ Centro de Investigaciones Energéticas, Medioambientales y Tecnológicas (CIEMAT), Madrid, Spain

${ }_{40}^{39}$ Instituto de Física, UFRGS, Caixa Postal 15051, Porto Alegre, RS—91501-970, Brazil

${ }^{40}$ Argonne National Laboratory, 9700 South Cass Avenue, Lemont, IL 60439, USA

${ }^{41}$ Department of Physics, Stanford University, 382 Via Pueblo Mall, Stanford, CA 94305, USA

Received 2016 March 6; revised 2016 October 13; accepted 2016 October 30; published 2017 January 6
}

\section{ABSTRACT}

We search for excess $\gamma$-ray emission coincident with the positions of confirmed and candidate Milky Way satellite galaxies using six years of data from the Fermi Large Area Telescope (LAT). Our sample of 45 stellar systems includes 28 kinematically confirmed dark-matter-dominated dwarf spheroidal galaxies (dSphs) and 17 recently discovered systems that have photometric characteristics consistent with the population of known dSphs. For each of these targets, the relative predicted $\gamma$-ray flux due to dark matter annihilation is taken from kinematic analysis if available, and estimated from a distance-based scaling relation otherwise, assuming that the 
stellar systems are DM-dominated dSphs. LAT data coincident with four of the newly discovered targets show a slight preference (each $\sim 2 \sigma$ local) for $\gamma$-ray emission in excess of the background. However, the ensemble of derived $\gamma$-ray flux upper limits for individual targets is consistent with the expectation from analyzing random blank-sky regions, and a combined analysis of the population of stellar systems yields no globally significant excess (global significance $<1 \sigma$ ). Our analysis has increased sensitivity compared to the analysis of 15 confirmed dSphs by Ackermann et al. The observed constraints on the DM annihilation cross section are statistically consistent with the background expectation, improving by a factor of $\sim 2$ for large DM masses $\left(m_{\mathrm{DM}, b \bar{b}} \gtrsim 1 \mathrm{TeV}\right.$ and $\left.m_{\mathrm{DM}, \tau^{+} \tau^{-}} \gtrsim 70 \mathrm{GeV}\right)$ and weakening by a factor of $\sim 1.5$ at lower masses relative to previously observed limits.

Key words: dark matter - galaxies: dwarf - gamma rays: galaxies

\section{INTRODUCTION}

Astrophysical evidence suggests that non-baryonic cold dark matter (DM) constitutes $\sim 84 \%$ of the matter density of the universe (Planck Collaboration 2015). Many particle DM candidates, such as weakly interacting massive particles (WIMPs), are predicted to annihilate or decay into energetic Standard Model particles (e.g., Bertone et al. 2005; Feng 2010). Depending on the DM particle mass and annihilation cross section or decay rate, these interactions may produce $\gamma$ rays detectable by instruments such as the Fermi Large Area Telescope (LAT), which is sensitive to $\gamma$ rays in the range from $20 \mathrm{MeV}$ to $>300 \mathrm{GeV}$ (Atwood et al. 2009). Milky Way dwarf spheroidal satellite galaxies (dSphs) are excellent targets to search for $\gamma$ rays produced from DM annihilation due to their proximity, their large DM density, and the absence of observational evidence for non-thermal astrophysical processes that produce $\gamma$ rays (e.g., Evans et al. 2004; Baltz et al. 2008).

The expected $\gamma$-ray flux from DM annihilation is

$$
\begin{aligned}
\phi\left(\Delta \Omega, E_{\min }, E_{\max }\right)= & \underbrace{\frac{1}{4 \pi} \frac{\langle\sigma v\rangle}{2 m_{\mathrm{DM}}^{2}} \int_{E_{\min }}^{E_{\max }} \frac{d N_{\gamma}}{d E_{\gamma}} \mathrm{d} E_{\gamma}}_{\text {particle physics }} \\
& \times \underbrace{\int_{E_{\min }} \int_{\Delta \Omega} \int_{\text {l.o.s. }} \rho_{\mathrm{DM}}^{2}(\boldsymbol{r}(l)) d l d \Omega}_{\text {Jfactor }},
\end{aligned}
$$

where $\langle\sigma v\rangle$ is the velocity-averaged DM annihilation cross section, $m_{\mathrm{DM}}$ is the DM particle mass, and $\frac{d N_{\gamma}}{d E}$ is the differential $\gamma$-ray photon counts spectrum summed over all final states. The "J-factor" is the square of the DM density $(\rho)$ as a function of position $\boldsymbol{l}$ integrated along the light-of-sight (l.o.s.) in the region of interest (ROI), and $\Delta \Omega$ denotes the solid angle over which the $J$-factor is calculated (Gondolo et al. 2004).

The $J$-factors of dSphs can be inferred from the measured velocities of their member stars (e.g., Simon \& Geha 2007; Walker et al. 2009). While the $J$-factors of individual dSphs are several orders of magnitude smaller than that of the Galactic center, observations of individual dSphs can be combined to increase the sensitivity to a DM annihilation signal while simultaneously reducing the impact of systematic uncertainties for individual dSphs. In addition, observations of the dSphs provide an important independent test of DM interpretations of the $\gamma$-ray excess associated with the Galactic center (GCE; Gordon \& Macias 2013; Abazajian et al. 2014; Calore et al. 2015; Ajello et al. 2016; Daylan et al. 2016).

Many groups have searched for excess $\gamma$ rays associated with dSphs using LAT data and have reported constraints on DM annihilation that are competitive with other DM targets such as the Galactic center (e.g., Abdo et al. 2010; Ackermann et al. 2011; Geringer-Sameth \& Koushiappas 2011; Mazziotta et al. 2012; Geringer-Sameth et al. 2015b, 2015c; Hooper \& Linden 2015; Li et al. 2016). For example, the combined likelihood analysis of $15 \mathrm{dSphs}$ with six years of LAT Pass 8 data by Ackermann et al. (2015b) excludes DM particles with masses $\lesssim 100 \mathrm{GeV}$ annihilating with the canonical thermal relic cross section via quark or $\tau$-lepton channels. That work used only dSphs with spectoscopically determined $J$-factors.

In 2015, a combination of on-going wide-field optical imaging surveys and a re-analysis of Sloan Digital Sky Survey (SDSS; York et al. 2000) data revealed more than 20 new satellite systems (Bechtol et al. 2015; Drlica-Wagner et al. 2015b; Kim et al. 2015a; Kim \& Jerjen 2015; Koposov et al. 2015a; Laevens et al. 2015a, 2015b; Martin et al. 2015b). The photometric characteristics of these new Milky Way satellites are consistent with previously known $\mathrm{dSphs}$, but are referred to as "dSph candidates" until their DM content is spectroscopically confirmed.

If the newly discovered systems are confirmed as DMdominated dSphs, they represent important new targets in the search for $\gamma$ rays from DM annihilation. This paper follows on the work of Drlica-Wagner et al. (2015a, hereafter DW15), who analyzed satellites discovered in the first year (Y1) of the Dark Energy Survey (DES; DES Collaboration 2005). Here, we perform a comprehensive Fermi-LAT $\gamma$-ray analysis of all confirmed and candidate dSphs. This target sample includes 15 additional dSph candidates found in year two (Y2) of DES and other surveys. In total, our sample comprises 45 confirmed and candidate dSphs (Section 2). We find slight ( $\sim 2 \sigma$ local) excesses of $\gamma$ rays coincident with four of the new targets (Section 3). Spectroscopic observations are needed to measure the dynamical masses and associated $J$-factors of the new systems. For recently discovered dSph candidates that lack spectroscopic observations, we use a simple scaling relation to predict $J$-factors based on photometric data alone (Section 4). In Section 5 we perform a combined analysis of the population of confirmed and candidate $\mathrm{dSphs}$ and find no globally significant excess associated with the ensemble of targets. We therefore present constraints on the DM annihilation cross section derived from the population of confirmed and candidate dSphs. In Section 6 we summarize our findings and conclude.

\section{TARGETS}

In 2015, wide-field optical imaging surveys enabled the discovery of more than 20 new Milky Way satellites having 
morphological characteristics similar to the known DMdominated dSphs. Each of these satellites was identified as a statistically significant arcminute-scale overdensity of resolved stars consistent with an old $(>10 \mathrm{Gyr})$ and metal-poor $(Z \sim 0.0002)$ simple stellar population. The basic structural characteristics of each stellar system (e.g., center position, heliocentric distance, and spatial extension) were inferred by fitting the spatial and color-magnitude distributions of probable member stars.

The majority of the recently announced Milky Way satellites were discovered in DES data collected with the Dark Energy Camera (DECam; Flaugher et al. 2015). Searches of the DES Y1 data by both the DES Collaboration and other groups led to the discovery of nine dSph candidates (Bechtol et al. 2015; Kim \& Jerjen 2015; Koposov et al. 2015a). Drlica-Wagner et al. (2015b) subsequently reported eight additional dSph candidates found in DES Y2 data. The compact stellar systems Kim 2 (Kim et al. 2015b) ${ }^{43}$ and DES 1 (Luque et al. 2016) are also present in the DES data; however, they have photometric properties that are more similar to lowluminosity outer-halo star clusters than to dSphs.

In addition to the objects found in DES data, several systems have recently been discovered in other surveys. Pegasus III was detected in archival SDSS data (Kim et al. 2015a) and later confirmed as a stellar overdensity with DECam. Hydra II was found serendipitously in DECam data taken for the Survey of the MAgellanic Stellar History (Martin et al. 2015b). Three additional $\mathrm{dSph}$ candidates were discovered in the PanSTARRS $13 \pi$ Survey: Triangulum II, Draco II, and Sagittarius II (Laevens et al. 2015a, 2015b). We note that several other systems have been discovered using Pan-STARRS 1, but due to their small sizes and/or measured kinematics, they are classified as globular clusters and are not considered in this work (Laevens et al. 2014, 2015a; Kirby et al. 2015b).

Thus far, six recently discovered systems have measured kinematics consistent with being DM-dominated dSphs: Reticulum II (Koposov et al. 2015b; Simon et al. 2015; Walker et al. 2015a), Horologium I (Koposov et al. 2015b), Hydra II (Kirby et al. 2015b), Draco II (Martin et al. 2015a), Triangulum II (Kirby et al. 2015a; Martin et al. 2016), and Tucana II (Walker et al. 2015b). J-factors have been derived for Reticulum II (Bonnivard et al. 2015b; Simon et al. 2015) and Tucana II (Walker et al. 2015b) from these kinematic data.

The dSphs are good targets for DM searches because their dynamical and chemical properties suggest the presence of large quantities of DM. In contrast, globular clusters have mass-to-light ratios of order unity. Low-luminosity stellar systems cannot be conclusively classified as dSphs or globular clusters without radial velocity measurements. However, dSphs are generally found to have larger physical half-light radii $\left(r_{1 / 2}\right)$ and lower surface brightnesses $(\mu)$ than globular clusters (Figure 1). Therefore, we used the photometric characteristics of the newly discovered systems to select those that are likely to be DM-dominated dSphs when spectroscopic measurements were unavailable.

For stellar systems with $M_{V} \lesssim-5$, DM-dominated dSphs have $r_{1 / 2} \gtrsim 100 \mathrm{pc}$, while globular clusters have $r_{1 / 2} \lesssim 20 \mathrm{pc}$. For fainter systems, the size distinction becomes less clear. The most compact kinematically classified dSph is Segue 1 (Geha

\footnotetext{
${ }^{43}$ Kim 2 was also identified as DES J2038-4610/Indus I by Bechtol et al. (2015) and Koposov et al. (2015a) slightly after its original discovery by Kim et al. (2015b).
}

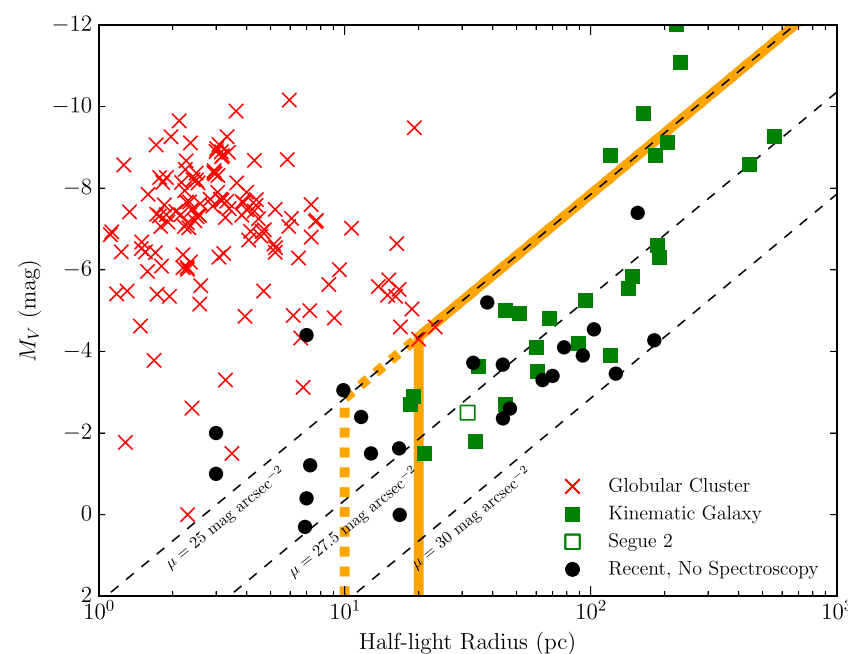

Figure 1. Absolute visual magnitude $\left(M_{V}\right)$ vs. physical half-light radius $\left(r_{1 / 2}\right)$ for dSphs and globular clusters. Globular clusters, which do not contain measurable DM within their visible stellar distribution, are marked with red crosses (Fadely et al. 2011; Harris 1996, 2010 edition). Spectroscopically confirmed DM-dominated dSphs are labeled with filled green squares. Segue 2 (open green square) has the chemical signatures of a dSph, but exhibits a lowvelocity dispersion (Kirby et al. 2013), and is therefore excluded from our target list. Milky Way satellites lacking spectroscopic observations are labeled with black filled circles. Thick orange lines indicate our target sample selection cuts on objects lacking spectroscopic data (see Section 5): nominal (solid; $r_{1 / 2}>20 \mathrm{pc}$ ) and inclusive (dashed; $r_{1 / 2}>10 \mathrm{pc}$ ). Black dashed lines indicate contours of constant surface brightness $(\mu)$.

et al. 2009; Simon et al. 2011), which has an azimuthally averaged half-light radius of $21 \mathrm{pc}$. On the other hand, the Palomar 13 globular cluster has a half-light radius of approximately $10 \mathrm{pc}$, and does not require DM to explain its measured velocity dispersion (Bradford et al. 2011). We note that recent spectroscopy of Draco II, which has an azimuthally averaged half-light radius of $16 \mathrm{pc}$, indicates a velocity dispersion $2.9 \pm 2.1 \mathrm{~km} \mathrm{~s}^{-1}$ and therefore is moderately likely to be DM dominated (Martin et al. 2015a). We inclusively selected new objects with $r_{1 / 2}>10 \mathrm{pc}$ and surface brightnesses $\mu>25$ mag $\operatorname{arcsec}^{-2}$.

Two confirmed globular clusters (Palomar 14 and Laevens 1) would pass our nominal selection criteria based on their physical size and luminosity $\left(r_{1 / 2} \sim 20 \mathrm{pc}, M_{V} \sim-4.5 \mathrm{mag}\right)$. However, Palomar 14 is kinematically determined to have a mass-to-light ratio near unity (Jordi et al. 2009), and the relatively large metallicity and low metallicity dispersion of Laevens 1 is more similar to globular clusters (Kirby et al. 2013). Therefore, we do not include these two systems in our analysis.

In Table 1 we summarize the characteristics of confirmed and candidate $\mathrm{dSphs}$ considered in this work. This table is divided into three sections: (1) systems that are kinematically determined to be DM-dominated dSphs, (2) systems with photometric characteristics consistent with known dSphs, and (3) systems with small physical sizes (10 pc $\left.<r_{1 / 2}<20 \mathrm{pc}\right)$ and ambiguous classifications (see Figure 1). Due to small stellar samples and/or complicated kinematics, several kinematically confirmed dSphs lack spectroscopically measured $J$-factors.

Several Milky Way satellites are not considered in this analysis. For instance, the Sagittarius and Canis Major dSphs are excluded because: (1) they reside at low Galactic latitude 
Table 1

Confirmed and Candidate Dwarf Galaxies

\begin{tabular}{|c|c|c|c|c|c|c|c|}
\hline (1) & (2) & (3) & (4) & $(5)$ & (6) & (7) & (8) \\
\hline Name & $\begin{array}{c}l, b \\
\text { (deg, deg) }\end{array}$ & $\begin{array}{l}\text { Distance } \\
\text { (kpc) }\end{array}$ & $\begin{array}{l}r_{1 / 2} \\
(\mathrm{pc})\end{array}$ & $\begin{array}{c}M_{V} \\
(\mathrm{mag})\end{array}$ & $\begin{array}{c}\log _{10}\left(J_{\text {meas }}\right) \\
\log _{10}\left(\mathrm{GeV}^{2} \mathrm{~cm}^{-5}\right)\end{array}$ & $\begin{array}{c}\log _{10}\left(J_{\text {pred }}\right) \\
\log _{10}\left(\mathrm{GeV}^{2} \mathrm{~cm}^{-5}\right)\end{array}$ & Sample \\
\hline \multicolumn{8}{|c|}{ Kinematically Confirmed Galaxies } \\
\hline Boötes I* & $358.08,69.62$ & 66 & 189 & -6.3 & $18.2 \pm 0.4$ & 18.5 & I, N, C \\
\hline Boötes II & $353.69,68.87$ & 42 & 46 & -2.7 & $\ldots$ & 18.9 & $\mathrm{I}, \mathrm{N}, \mathrm{C}$ \\
\hline Boötes III & $35.41,75.35$ & 47 & $\ldots$ & -5.8 & $\ldots$ & 18.8 & $\mathrm{I}, \mathrm{N}$ \\
\hline Canes Venatici I & $74.31,79.82$ & 218 & 441 & -8.6 & $17.4 \pm 0.3$ & 17.4 & $\mathrm{I}, \mathrm{N}, \mathrm{C}$ \\
\hline Canes Venatici II & $113.58,82.70$ & 160 & 52 & -4.9 & $17.6 \pm 0.4$ & 17.7 & $\mathrm{I}, \mathrm{N}, \mathrm{C}$ \\
\hline Carina* ${ }^{*}$ & $260.11,-22.22$ & 105 & 205 & -9.1 & $17.9 \pm 0.1$ & 18.1 & $\mathrm{I}, \mathrm{N}, \mathrm{C}$ \\
\hline Coma Berenices* & $241.89,83.61$ & 44 & 60 & -4.1 & $19.0 \pm 0.4$ & 18.8 & $\mathrm{I}, \mathrm{N}, \mathrm{C}$ \\
\hline Draco* & $86.37,34.72$ & 76 & 184 & -8.8 & $18.8 \pm 0.1$ & 18.3 & $\mathrm{I}, \mathrm{N}, \mathrm{C}$ \\
\hline Draco II & $98.29,42.88$ & 24 & 16 & -2.9 & $\ldots$ & 19.3 & $\mathrm{I}, \mathrm{N}, \mathrm{C}$ \\
\hline Fornax ${ }^{*}$ & $237.10,-65.65$ & 147 & 594 & -13.4 & $17.8 \pm 0.1$ & 17.8 & $\mathrm{I}, \mathrm{N}, \mathrm{C}$ \\
\hline Hercules* & $28.73,36.87$ & 132 & 187 & -6.6 & $16.9 \pm 0.7$ & 17.9 & $\mathrm{I}, \mathrm{N}, \mathrm{C}$ \\
\hline Horologium I & $271.38,-54.74$ & 87 & 61 & -3.5 & $\ldots$ & 18.2 & $\mathrm{I}, \mathrm{N}, \mathrm{C}$ \\
\hline Hydra II & $295.62,30.46$ & 134 & 66 & -4.8 & $\ldots$ & 17.8 & $\mathrm{I}, \mathrm{N}, \mathrm{C}$ \\
\hline Leo I & $225.99,49.11$ & 254 & 223 & -12.0 & $17.8 \pm 0.2$ & 17.3 & $\mathrm{I}, \mathrm{N}, \mathrm{C}$ \\
\hline Leo II* & $220.17,67.23$ & 233 & 164 & -9.8 & $18.0 \pm 0.2$ & 17.4 & $\mathrm{I}, \mathrm{N}, \mathrm{C}$ \\
\hline Leo $I^{*}$ & $265.44,56.51$ & 154 & 147 & -5.8 & $16.3 \pm 1.4$ & 17.7 & $\mathrm{I}, \mathrm{N}, \mathrm{C}$ \\
\hline Leo V & $261.86,58.54$ & 178 & 95 & -5.2 & $16.4 \pm 0.9$ & 17.6 & $\mathrm{I}, \mathrm{N}, \mathrm{C}$ \\
\hline Pisces II & $79.21,-47.11$ & 182 & 45 & -5.0 & $\ldots$ & 17.6 & $\mathrm{I}, \mathrm{N}, \mathrm{C}$ \\
\hline Reticulum II & $266.30,-49.74$ & 32 & 35 & -3.6 & $18.9 \pm 0.6$ & 19.1 & $\mathrm{I}, \mathrm{N}, \mathrm{C}$ \\
\hline Sculptor* & $287.53,-83.16$ & 86 & 233 & -11.1 & $18.5 \pm 0.1$ & 18.2 & $\mathrm{I}, \mathrm{N}, \mathrm{C}$ \\
\hline Segue $1^{*}$ & $220.48,50.43$ & 23 & 21 & -1.5 & $19.4 \pm 0.3$ & 19.4 & $\mathrm{I}, \mathrm{N}, \mathrm{C}$ \\
\hline Sextans* & $243.50,42.27$ & 86 & 561 & -9.3 & $17.5 \pm 0.2$ & 18.2 & $\mathrm{I}, \mathrm{N}, \mathrm{C}$ \\
\hline Triangulum II & $140.90,-23.82$ & 30 & 30 & -1.8 & $\ldots$ & 19.1 & $\mathrm{I}, \mathrm{N}, \mathrm{C}$ \\
\hline Tucana II & $328.04,-52.35$ & 58 & 120 & -3.9 & $\ldots$ & 18.6 & $\mathrm{I}, \mathrm{N}, \mathrm{C}$ \\
\hline Ursa Major I & $159.43,54.41$ & 97 & 143 & -5.5 & $17.9 \pm 0.5$ & 18.1 & $\mathrm{I}, \mathrm{N}, \mathrm{C}$ \\
\hline Ursa Major II ${ }^{*}$ & $152.46,37.44$ & 32 & 91 & -4.2 & $19.4 \pm 0.4$ & 19.1 & $\mathrm{I}, \mathrm{N}, \mathrm{C}$ \\
\hline Ursa Minor* & $104.97,44.80$ & 76 & 120 & -8.8 & $18.9 \pm 0.2$ & 18.3 & $\mathrm{I}, \mathrm{N}, \mathrm{C}$ \\
\hline Willman $1^{*}$ & $158.58,56.78$ & 38 & 19 & -2.7 & $\ldots$ & 18.9 & $\mathrm{I}, \mathrm{N}$ \\
\hline \multicolumn{8}{|c|}{ Likely Galaxies } \\
\hline Columba I & $231.62,-28.88$ & 182 & 101 & -4.5 & $\ldots$ & 17.6 & $\mathrm{I}, \mathrm{N}, \mathrm{C}$ \\
\hline Eridanus II & $249.78,-51.65$ & 331 & 156 & -7.4 & $\ldots$ & 17.1 & $\mathrm{I}, \mathrm{N}, \mathrm{C}$ \\
\hline Grus I & $338.68,-58.25$ & 120 & 60 & -3.4 & $\ldots$ & 17.9 & $\mathrm{I}, \mathrm{N}, \mathrm{C}$ \\
\hline Grus II & $351.14,-51.94$ & 53 & 93 & -3.9 & $\cdots$ & 18.7 & $\mathrm{I}, \mathrm{N}, \mathrm{C}$ \\
\hline Horologium II & $262.48,-54.14$ & 78 & 33 & -2.6 & $\ldots$ & 18.3 & $\mathrm{I}, \mathrm{N}, \mathrm{C}$ \\
\hline Indus II & $354.00,-37.40$ & 214 & 181 & -4.3 & $\ldots$ & 17.4 & $\mathrm{I}, \mathrm{N}, \mathrm{C}$ \\
\hline Pegasus III & $69.85,-41.81$ & 205 & 57 & -4.1 & $\ldots$ & 17.5 & $\mathrm{I}, \mathrm{N}, \mathrm{C}$ \\
\hline Phoenix II & $323.69,-59.74$ & 96 & 33 & -3.7 & $\ldots$ & 18.1 & $\mathrm{I}, \mathrm{N}, \mathrm{C}$ \\
\hline Pictor I & $257.29,-40.64$ & 126 & 44 & -3.7 & $\ldots$ & 17.9 & $\mathrm{I}, \mathrm{N}, \mathrm{C}$ \\
\hline Reticulum III & $273.88,-45.65$ & 92 & 64 & -3.3 & $\ldots$ & 18.2 & $\mathrm{I}, \mathrm{N}, \mathrm{C}$ \\
\hline Sagittarius II & $18.94,-22.90$ & 67 & 34 & -5.2 & $\ldots$ & 18.4 & $\mathrm{I}, \mathrm{N}, \mathrm{C}$ \\
\hline Tucana III & $315.38,-56.18$ & 25 & 44 & -2.4 & $\ldots$ & 19.3 & $\mathrm{I}, \mathrm{N}$ \\
\hline Tucana IV & $313.29,-55.29$ & 48 & 128 & -3.5 & $\ldots$ & 18.7 & $\mathrm{I}, \mathrm{N}, \mathrm{C}$ \\
\hline \multicolumn{8}{|c|}{ Ambiguous Systems } \\
\hline Cetus II & $156.47,-78.53$ & 30 & 17 & 0.0 & $\ldots$ & 19.1 & I \\
\hline Eridanus III & $274.95,-59.60$ & 96 & 12 & -2.4 & $\ldots$ & 18.1 & I \\
\hline Kim 2 & $347.16,-42.07$ & 105 & 12 & -1.5 & $\ldots$ & 18.1 & I \\
\hline Tucana V & $316.31,-51.89$ & 55 & 16 & -1.6 & $\ldots$ & 18.6 & I \\
\hline
\end{tabular}

Note. Milky Way satellite systems consistent with being dSphs. Horizontal lines divide systems that have been kinematically determined to be DM dominated (top), systems with photometry consistent with being dSphs (middle), and systems with small physical sizes populating an ambiguous region of the size-luminosity plane between dSphs and globular clusters (bottom). Columns represent (1) name of stellar system, (2) Galactic coordinates, (3) heliocentric distance, (4) azimuthally averaged half-light radius, (5) absolute visual magnitude, (6) measured $J$-factor derived from stellar kinematics by Geringer-Sameth et al. (2015a); Reticulum II value taken from Simon et al. (2015) (7) predicted $J$-factor from Equation (2) (8) composite sample membership (see Section 5): C = conservative, N = nominal, $\mathrm{I}=$ inclusive. Targets used in the combined limits from Ackermann et al. (2015b) are marked with asterisks.

$(b=-14.2$ and $b=-8.0$, respectively) where the diffuse Galactic $\gamma$-ray foreground emission presents both statistical and systematic challenges, and (2) they show strong evidence of tidal disruption, making accurate determination of their DM masses difficult (Martin et al. 2004; Frinchaboy et al. 2012). In spite of these obstacles, the proximity $(26 \mathrm{kpc}$ and $7 \mathrm{kpc}$, 

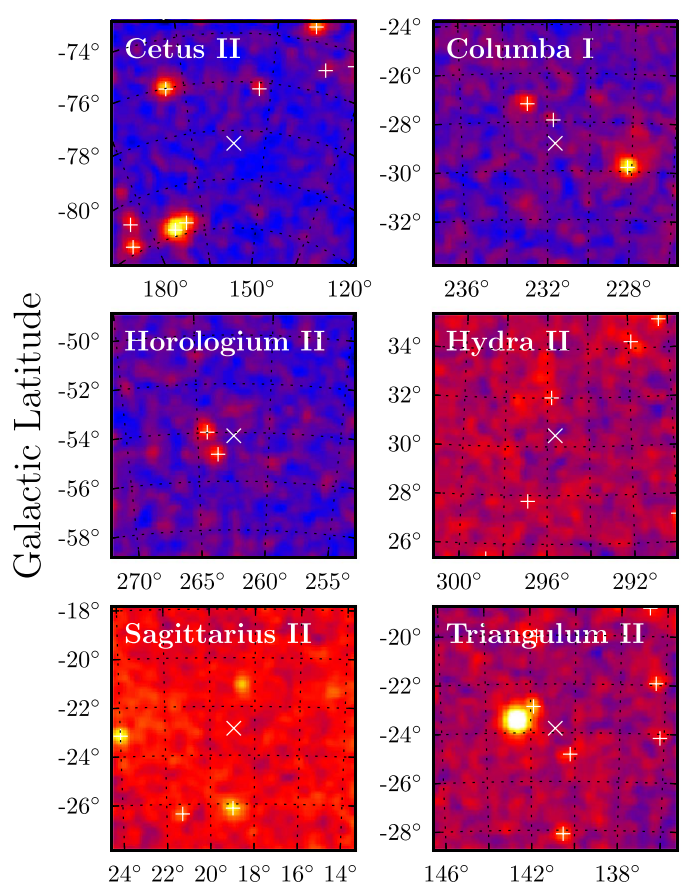

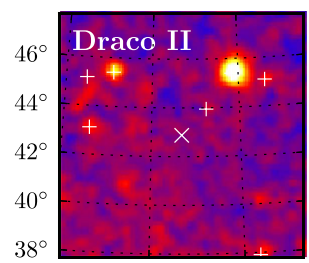

$100^{\circ} \quad 95^{\circ}$
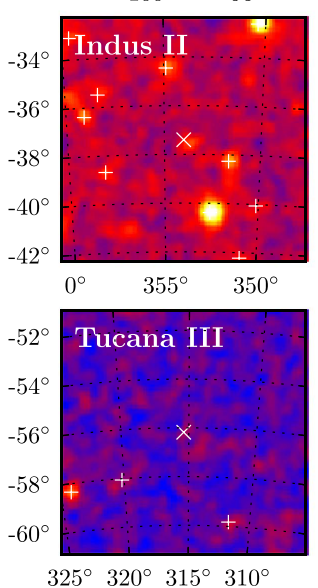

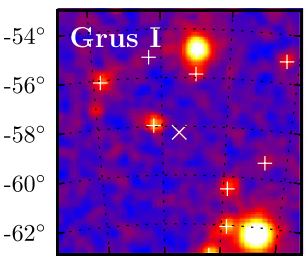

$345^{\circ} 340^{\circ} 335^{\circ} 330^{\circ}$
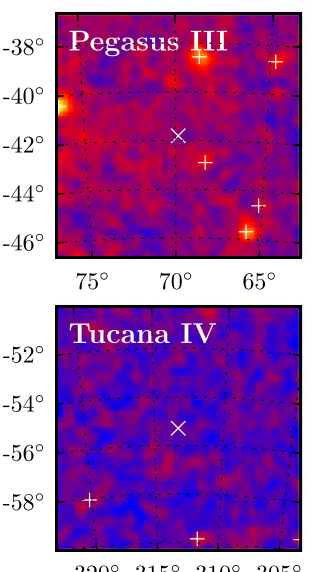

$320^{\circ} 315^{\circ} 310^{\circ} 305^{\circ}$
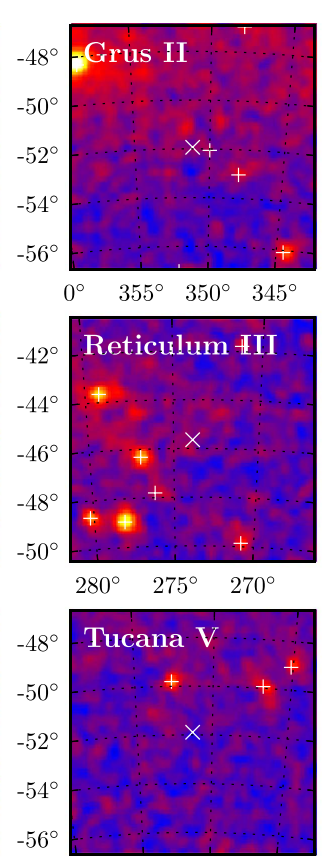

$325^{\circ} 320^{\circ} 315^{\circ} 310^{\circ}$

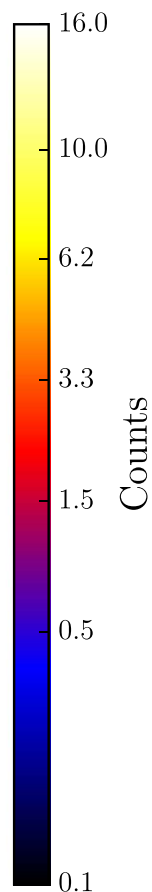

Galactic Longitude

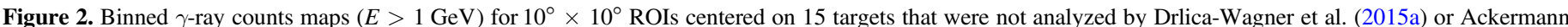

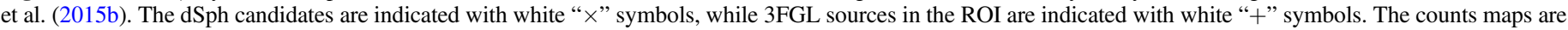
binned in $0.1 \times 0.1$ spatial pixels and smoothed with a 0.25 Gaussian kernel.

respectively) and large velocity dispersions of these two systems make them promising targets for dedicated individual study.

Finally, we exclude Segue 2 from our target list. Spectroscopic measurements show that Segue 2 has a large metallicity dispersion characteristic of dSphs, but the upper bound on its velocity dispersion, $\sigma_{v}<2.2 \mathrm{~km} \mathrm{~s}^{-1}$, implies a mass-to-light ratio within the half-light radius, $\left(M / L_{V}\right)_{1 / 2}<360 M_{\odot} / L_{\odot}$, lower than that of other comparably luminous dSphs (Kirby et al. 2013). As shown in Figure 1, Segue 2 is situated within the locus of DM-dominated dSphs according to its photometric properties, and therefore provides a cautionary example of a system that might not follow the scaling relation described in Section 4, which assumes a common value for the central DM density of dSphs.

\section{LAT ANALYSIS}

We analyzed $\gamma$-ray data coincident with our targets using the same analysis procedure and data set described in Ackermann et al. (2015b). We briefly review the details of the analysis here for completeness. Our data set consisted of six years of LAT data (2008 August 4 to 2014 August 5) in the energy range from $500 \mathrm{MeV}$ to $500 \mathrm{GeV}$ passing the P8R2 SOURCE event class selections. We rejected events with zenith angles greater than $100^{\circ}$ to remove $\gamma$ rays produced by cosmic-ray interactions in the Earth's atmosphere. Additionally, events from time intervals around bright $\gamma$-ray bursts and solar flares were removed using the same procedure as the third LAT source catalog (3FGL; Acero et al. 2015). To analyze the targets in Table 1 , we used $10^{\circ} \times 10^{\circ}$ ROIs centered on each target. In Figure 2 we show $\gamma$-ray counts maps for 15 systems that were not previously analyzed by DW15 or Ackermann et al. (2015b). Data reduction was performed using the Fermi ScienceTools ${ }^{44}$ version 10-01-01 and the P8R2_SOURCE_V6 instrument response functions. ${ }^{45}$

To search for $\gamma$-ray emission coincident with our targets in excess of the local background expectation, we performed a binned maximum-likelihood analysis in 24 logarithmically spaced energy bins and 0.1 spatial pixels. Data were partitioned into four point-spread function event types, which were combined in a joint likelihood function when fitting each ROI (Ackermann et al. 2015b). We modeled the Galactic diffuse emission with the standard LAT interstellar emission model (gll_iem_v06.fits) recommended for analysis of the Pass 8 data. ${ }^{46}$ Additionally, we modeled extragalactic $\gamma$-ray emission and residual charged particle contamination with an isotropic model fit to the Pass 8 data. Point sources from the 3FGL catalog within $15^{\circ}$ of the ROI center were included in the background model. The flux normalizations of the Galactic, isotropic, and $3 \mathrm{FGL}$ catalog sources within the $10^{\circ} \times 10^{\circ} \mathrm{ROI}$ were fit simultaneously over the broadband energy range from $500 \mathrm{MeV}$ to $500 \mathrm{GeV}$. The spectral parameters of all other background components were fixed to their nominal values during the fit. Following DW15, we enabled the energy dispersion correction in our fits for all components except the Galactic diffuse emission model and the isotropic model. The flux normalizations of the background sources were insensitive to the inclusion of a putative power-law source at the locations of the targets. Each ROI was found to be well described by the

\footnotetext{
${ }^{44}$ http://fermi.gsfc.nasa.gov/ssc/data/analysis/software/

45 http://www.slac.stanford.edu/exp/glast/groups/canda/lat_ Performance.htm

46 http://fermi.gsfc.nasa.gov/ssc/data/access/lat/BackgroundModels.html
} 


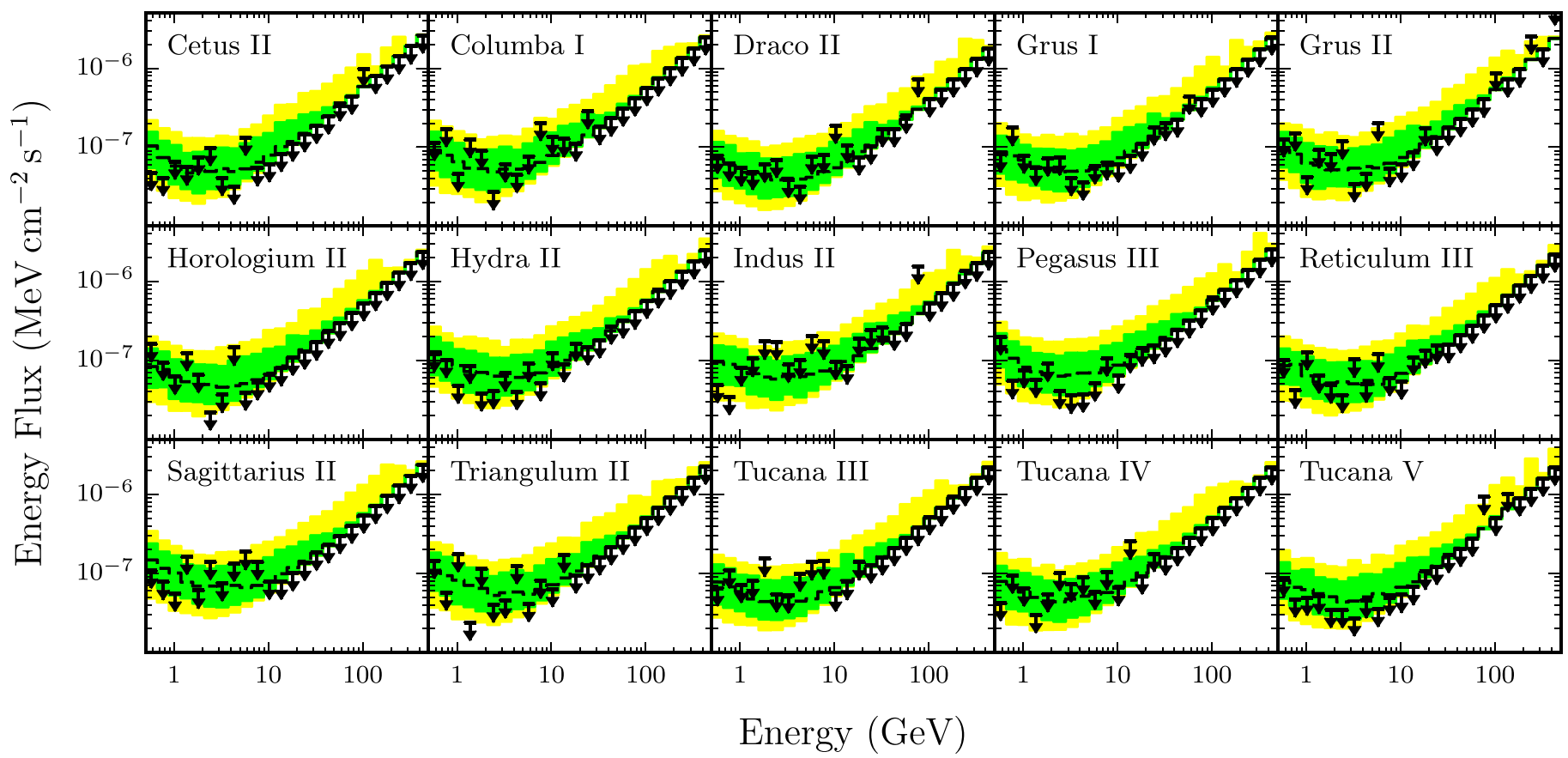

Figure 3. Bin-by-bin integrated energy-flux upper limits at $95 \%$ confidence level assuming a point-like model for the 15 targets in Figure 2 . The median expected sensitivity is shown by the dashed black line while the $68 \%$ and $95 \%$ containment regions are indicated by the green and yellow bands, respectively. The expected sensitivity and containment regions are derived from 300 Monte Carlo simulations of the $\gamma$-ray background in the regions surrounding each respective target.

background model with no significant $(>3 \sigma)$ residuals associated with the target locations.

In this analysis we modeled all 45 confirmed and candidate dSphs in Table 1 as point-like sources. This differs from the analysis of Ackermann et al. (2015b) where some targets were modeled as extended sources. This choice was motivated by a desire to have a consistent analysis across the targets and the fact that the physical sizes of the DM halos surrounding the dSph candidates are not well constrained (we further investigate the impact of this choice in Section 5). We fit for excess $\gamma$-ray emission above the background associated with each target in each energy bin separately to derive upper limits on the flux that are independent of the choice of spectral model. Within each bin, we model the putative $\mathrm{dSph}$ source with a power-law spectral model $\left(d N / d E \propto E^{-\Gamma}\right)$ with spectral index $\Gamma=2$ (Ackermann et al. 2014, 2015b). In Figure 3 we show the bin-by-bin integrated energy-flux upper limits at $95 \%$ confidence level for $15 \mathrm{dSph}$ candidates not included in DW15 or Ackermann et al. (2015b). We generate 68\% and 95\% containment bands for the bin-by-bin limits from 300 Monte Carlo simulations of the local $\gamma$-ray background in the region of each dSph using the Fermi ScienceTool, gtobssim. We use simulations to account for local variations in the diffuse $\gamma$-ray background between the individual $\mathrm{dSphs}$ (Ackermann et al. 2014).

The $10^{\circ} \times 10^{\circ}$ ROIs of several targets overlap, and we investigated possible correlations between the normalization of the putative sources. The two targets with the smallest angular separation $(\sim 1.5)$ are Tucana III and Tucana IV. We simultaneously fit the normalizations of the Galactic diffuse emission model, Tucana III, and Tucana IV in several energy bins, and found the magnitude of the correlation factor between the normalizations of the two dSph candidates to be $<0.1$. The LAT is certainly capable of resolving more closely spaced sources (see Figure 13 of Acero et al. 2015), and our result is consistent with that of Carlson et al. (2015), who studied the correlation between dSph targets and nearby unresolved sources.

To maximize the sensitivity to specific DM spectral models, the Poisson likelihoods from each bin were combined to form broadband likelihoods for different DM annihilation channels and masses. We tested for excess $\gamma$-ray emission consistent with two representative DM annihilation channels (i.e., $b \bar{b}$ and $\tau^{+} \tau^{-}$), and scanned a range of DM particle masses in six steps per decade from $2 \mathrm{GeV}$ to $10 \mathrm{TeV}$ (when kinematically allowed). The spectra were obtained from DMFit based on PYTHIA 8.165 using the Fermi ScienceTools (Jeltema \& Profumo 2008; Ackermann et al. 2014, 2015b). We calculated a test statistic (TS) for $\gamma$-ray source detection from the logarithm of the likelihood ratio when fitting the ROI with and without the putative dSph source (see Equation (6) in Ackermann et al. 2015b). We note that the TS of an individual target does not depend on the assumed $J$-factor; however, both the $J$-factor and its uncertainty affect the DM interpretation. No significant excess $\gamma$-ray emission above the background was observed coincident with any of the targets for any of the DM masses or channels tested. Several of the targets show slight $(<2.5 \sigma$ local $)$ excesses with respect to the background and are discussed further.

In Figure 4 we show the TS values from the likelihood analysis of each target as a function of annihilation channel and DM mass. We also show the one-sided 84\% and 97.5\% containment bands from performing our analysis on blank-sky locations. ${ }^{47}$ There are four targets with maximum TS values

\footnotetext{
47 The blank-sky locations used to calibrate detection significance are randomly distributed at Galactic latitudes $|b|>30^{\circ}$. The average diffuse background intensity in this region is within $5 \%$ of the average diffuse background intensity in the 45 target ROIs. The incidence of unmodeled point sources is expected to be similar at blank-sky locations and in the target ROIs because the $\gamma$-ray sources detected at high Galactic latitudes are approximately isotropically distributed.
} 

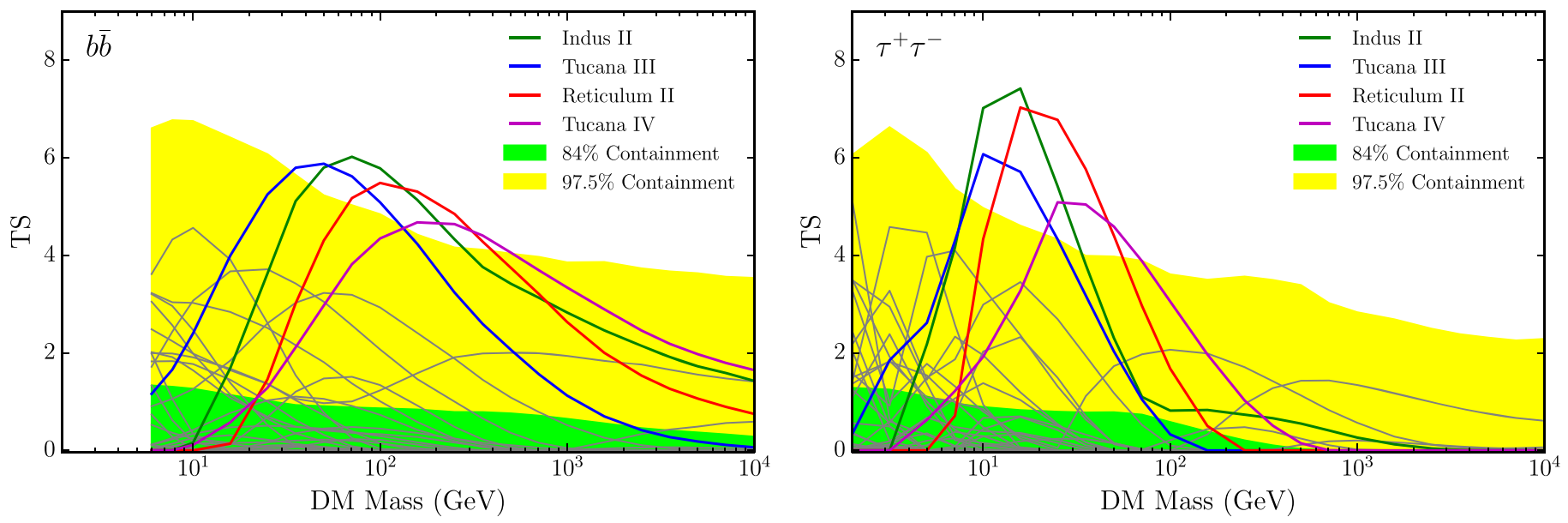

Figure 4. Local detection significance, expressed as a log-likelihood test statistic (TS), from the broadband analysis of each target in Table 1 assuming DM annihilation through the $b \bar{b}$ (left) or $\tau^{+} \tau^{-}$(right) channels. The bands represent the local one-sided $84 \%$ (green) and 97.5\% (yellow) containment regions derived from 300 random sets of 45 blank-sky locations. Curves corresponding to targets with peak significance larger than the local $95 \%$ expectation from blank-sky regions are explicitly colored and labeled, while other targets are shown in gray.

Table 2

Targets with the Largest Excesses above Background

\begin{tabular}{|c|c|c|c|c|c|c|}
\hline (1) & (2) & (3) & (4) & (5) & (6) & (7) \\
\hline Name & Channel & Mass $(\mathrm{GeV})$ & TS & $p_{\text {local }}$ & $p_{\text {target }}$ & $p_{\text {sample }}$ \\
\hline Indus II & $\tau^{+} \tau^{-}$ & 15.8 & 7.4 & $0.01(2.3 \sigma)$ & $0.04(1.7 \sigma)$ & $0.84(-1.0 \sigma)$ \\
\hline Reticulum II & $\tau^{+} \tau^{-}$ & 15.8 & 7.0 & $0.01(2.3 \sigma)$ & $0.05(1.7 \sigma)$ & $0.88(-1.2 \sigma)$ \\
\hline Tucana III & $\tau^{+} \tau^{-}$ & 10.0 & 6.1 & $0.02(2.1 \sigma)$ & $0.06(1.5 \sigma)$ & $0.94(-1.6 \sigma)$ \\
\hline Tucana IV & $\tau^{+} \tau^{-}$ & 25.0 & 5.1 & $0.02(2.1 \sigma)$ & $0.09(1.3 \sigma)$ & $0.98(-2.1 \sigma)$ \\
\hline
\end{tabular}

Note. (1) Target name, (2) best-fit DM annihilation channel, (3) best-fit DM particle mass, (4) highest TS value, (5) local $p$-value calibrated from random blank regions, (6) target $p$-value applying a trials factor from testing multiple DM annihilation spectra, (7) sample $p$-value applying an additional trials factor from analyzing 45 targets. The Gaussian significance associated with each $p$-value is given in parentheses. More details can be found in Section 3.

exceeding the local 95\% containment contours from an analysis of blank-sky regions: Indus II, Reticulum II, Tucana III, and Tucana IV. We note that other independent analyses have found significant $\left(p_{\text {local }}>3 \sigma\right)$ emission from Reticulum II (Geringer-Sameth et al. 2015c; Hooper \& Linden 2015). The $p_{\text {local }}$ of Reticulum II in this analysis is smaller mostly due to the use of the Pass 8 dataset as opposed to the Pass 7 Reprocessed dataset.

All four targets in Table 2 have TS $<7.5$ when fit over the broad-band energy range with any DM spectral model (TS $<4$ when fit with a single $\Gamma=2$ power-law spectral model). The best-fit masses, channels, and significances of these excesses are shown in Table 2. We quote three $p$-values: (1) the local $p$ value at the best-fit DM mass and channel, $p_{\text {local }}$, (2) the $p$ value per target, $p_{\text {target }}$, which takes into account the trials factor from scanning multiple DM masses and channels, and (3) the sample $p$-value, $p_{\text {sample }}$, which includes an additional trials factor from analyzing 45 target locations. $p_{\text {local }}$ and $p_{\text {target }}$ are empirically determined with respect to 300 sets of 45 blanksky locations (Ackermann et al. 2014). For a particular target, the null distribution for $p_{\text {local }}$ is the distribution of TS evaluated at the best-fit DM mass and channel, whereas the null distribution for $p_{\text {target }}$ is the distribution of the maximum TS over all considered DM masses and channels at each blank-sky location. We use the TS distribution from fits in blank-sky locations to account for the effect of unmodeled components of the $\gamma$-ray sky such as unresolved point sources (see Figure 6 of the supplemental material for Ackermann et al. 2015b).
In the background-only case without a DM annihilation signal, analyzing 45 targets will yield four or more targets with detection significances exceeding the $p_{\text {target }}$ values in Table 2 $45 \%$ of the time. However, this naive calculation treats each target equally, whereas the predicted $\gamma$-ray flux from DM annihilation is proportional to the $J$-factor. In Section 5, we describe a combined analysis that weights the targets by their $J$-factors and links the spectral model (DM mass and annihilation channel) across targets, and thereby enhances the sensitivity to a collective DM signal from the population of Milky Way satellites.

No 3FGL sources are located within $1^{\circ}$ of any of the four systems mentioned above. We also investigated associations with sources observed at other wavelengths that are potential $\gamma$ ray emitters in the BZCAT (Massaro et al. 2009), CRATES (Healey et al. 2007), CGraBS (Healey et al. 2008), PMN (Wright et al. 1994), and WISE blazar candidate (D'Abrusco et al. 2014) catalogs. We find two sources from the PMN catalog, PMN J0335-5406 and PMN J0335-5352, within 15' of Reticulum II. The first of these, PMN J0335-5406, has a relatively large flux at low frequency $(225 \mathrm{mJy}$ at $843 \mathrm{MHz})$ and a fairly hard radio spectral index $(\Gamma \sim 0.7)$, making it a possible $\gamma$-ray emitter (Ackermann et al. 2015a). In addition, the infrared colors of PMN J0335-5406 measured with WISE are consistent with other known $\gamma$-ray emitting blazars (Massaro et al. 2011). However, we note that this source is relatively faint in the optical/near-infrared, having $z \gtrsim 23 \mathrm{mag}$ in the DES imaging. The second source, PMN J0335-5352, has a smaller radio flux and seems unlikely to be associated 


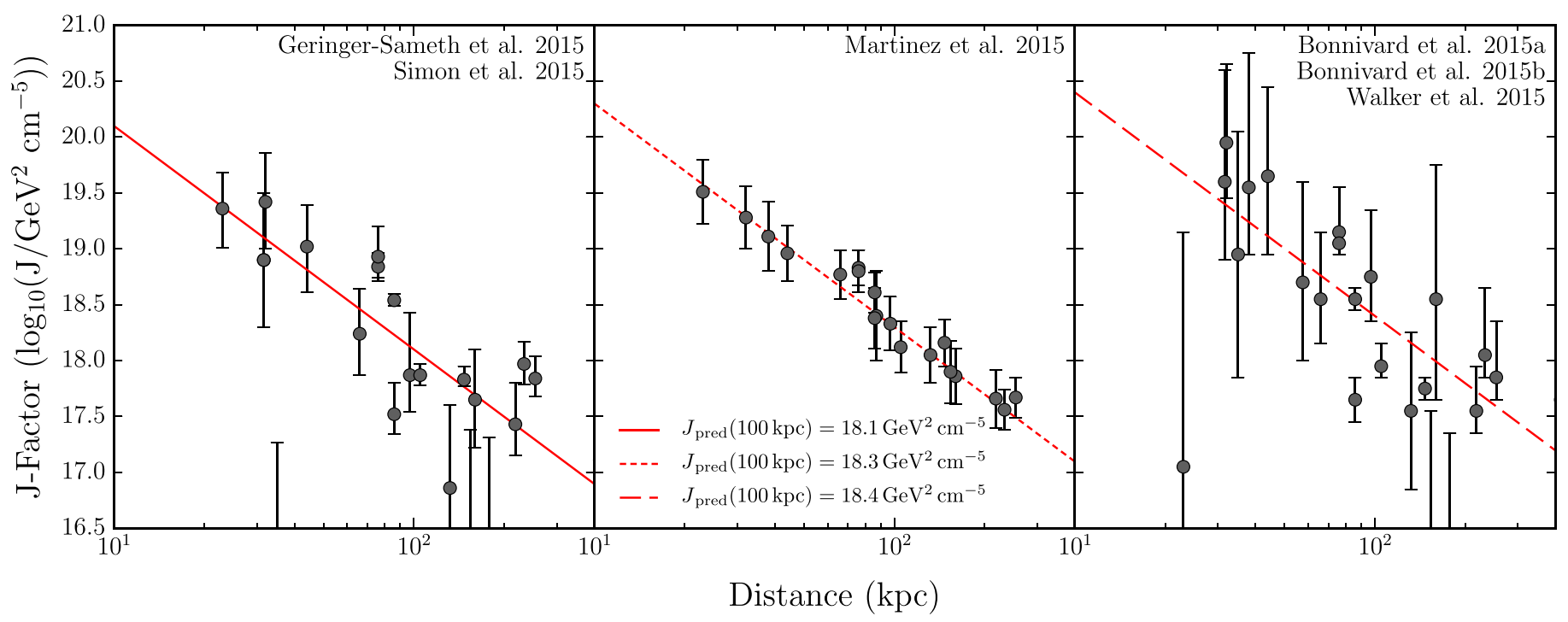

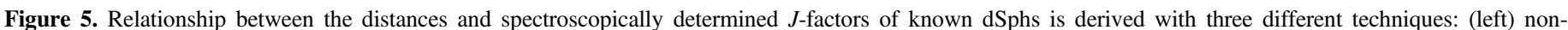

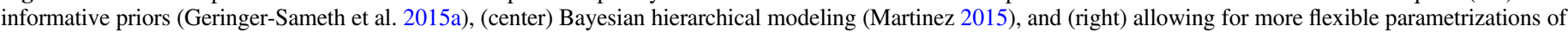

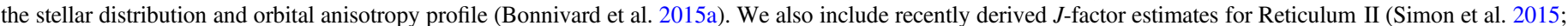

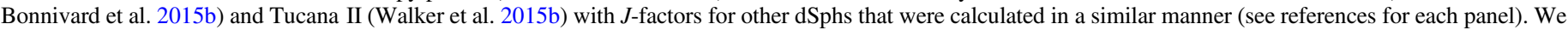

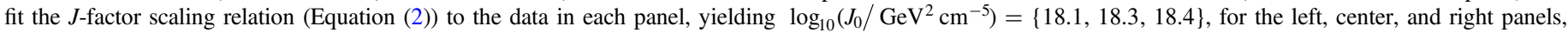
respectively; these relationships are plotted as solid, short dashed, and long dashed red lines.

with a $\gamma$-ray emitting blazar. We additionally find the source PMN J0003-6059 located 10' from Tucana IV, but due to the lack of multifrequency measurements it is unclear whether it is a potential $\gamma$-ray emitter.

\section{ESTIMATING $J$-FACTORS}

An estimate of the $J$-factor is necessary to convert a $\gamma$-ray flux upper limit into a constraint on the DM annihilation cross section (Equation (1)). The $J$-factor depends on both the DM density profile and distance. Distances can be determined from the photometric data using the characteristic absolute magnitude of the main-sequence turn-off and/or horizontal branch in old, metal-poor stellar populations. On the other hand, measurement of the DM mass requires spectroscopic observations to determine the radial velocities of member stars. The classical dSphs discovered prior to SDSS have measured velocity dispersions in the range $\sim 6-11 \mathrm{~km} \mathrm{~s}^{-1}$, and the ultrafaint dSphs discovered by SDSS have velocity dispersions in the range $\sim 2-6 \mathrm{~km} \mathrm{~s}^{-1}$. Similarly, the six new systems recently confirmed as $\mathrm{dSphs}$ have velocity dispersions in the range from $2.9-8.6 \mathrm{~km} \mathrm{~s}^{-1}$.

The known dSphs have similar central DM densities despite a wide spread in optical luminosity (Strigari et al. 2008). The similarity in the central DM density of the dSphs causes their $J$-factors to scale approximately as the inverse square of their distances. In Figure 5, we show that a simple scaling relationship between $J$-factor and distance can be clearly seen in the $J$-factors derived by several groups (i.e., Bonnivard et al. 2015a; Geringer-Sameth et al. 2015a; Martinez 2015). For each set of $J$-factor measurements, the intrinsic scatter relative to the proposed scaling relationship appears to be smaller than the average measurement uncertainty.

Following DW15, we assume that the new stellar systems occupy similar DM halos to the population of known dSphs, and we predict the $J$-factors of the new systems from their distances. This assumption is necessary to convert the $\gamma$-ray flux limits to DM annihilation cross section constraints since most of the newly discovered systems have not yet been observed spectroscopically. We do not expect globular clusters to follow the same scaling relation, since their observed velocity dispersions imply that they do not contain DM.

For each candidate we calculated a predicted $J$-factor using the procedure developed in DW15. Our scaling relationship is

$$
\log _{10}\left(\frac{J_{\text {pred }}}{J_{0}}\right)=-2 \log _{10}\left(\frac{D}{100 \mathrm{kpc}}\right),
$$

where $D$ is the heliocentric distance of the $\mathrm{dSph}$ candidate and $J_{0}$ is a scale factor derived from a fit to spectroscopic data (Figure 5). In contrast to DW15, we derived our nominal scale factor, $J_{0}=18.1 \mathrm{GeV}^{2} \mathrm{~cm}^{-5}$, using the spectroscopic $J$-factors from Geringer-Sameth et al. (2015b) as opposed to those from Martinez (2015). The two data sets give compatible results (see DW15); however, the $J$-factors derived by Geringer-Sameth et al. (2015b) rely on fewer assumptions about the population of dSphs and provide slightly more conservative estimates for the predicted $J$-factors. The predicted $J$-factor for each stellar system is shown in Table 1 .

In addition to predicting the value of the $J$-factor we approximate the uncertainty achievable with future radial velocity measurements. The uncertainty on the $J$-factor derived from spectroscopic observations depends on several factors, most importantly the number of stars for which radial velocities have been measured. For ultra-faint dSphs that are similar to the dSph candidates, spectra have been measured for 20-100 stars. Additional sources of uncertainty include the DM density profile and dynamical factors such as the velocity anisotropy of member stars. We consider characteristic $J$-factor uncertainties, $\log _{10} \sigma_{J}=\{0.4,0.6,0.8\}$ dex, for the newly discovered ultrafaint satellites lacking spectroscopically determined $J$-factors. Note that these uncertainties refer to characteristic measurement uncertainties on the $J$-factor for a typical dSph, and do not reflect any intrinsic scatter that may exist in a larger population of satellites. 

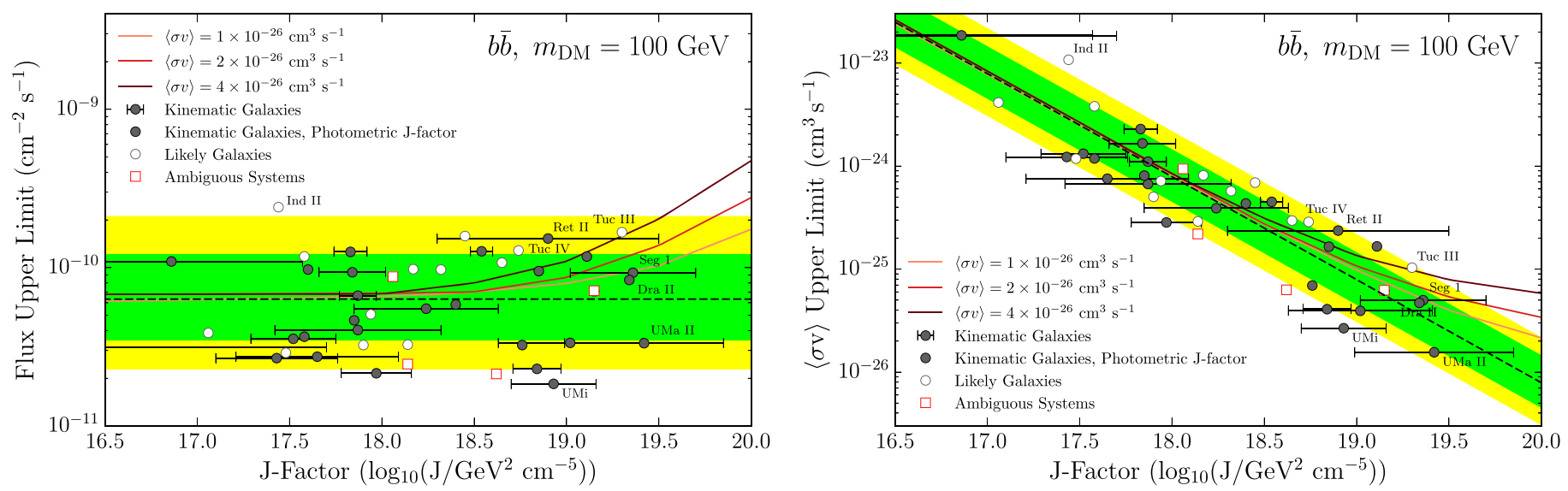

Figure 6. Upper limits on flux (left) and cross section (right) vs. $J$-factor. The points represent $J$-factors for each target estimated either from spectroscopy (filled circles with error bars) or from the scaling relation discussed in Section 4 (filled circles). The green and yellow shaded regions are the $68 \%$ and $95 \%$ containment regions for the blank-sky expectations, respectively. For comparison, the three solid lines show the median expected upper limits for DM annihilation with the given cross section. No significant deviation from the background-only expectation is observed.

We reiterate that this analysis assumes that the newly discovered systems are DM-dominated, similar to the known population of ultra-faint dSphs. Some of the more compact systems might actually be faint outer-halo star clusters. Some of the larger systems also may be subject to tidal stripping, in which case the distance-based estimation described above may not apply. Ongoing spectroscopic analyses seek to robustly determine the DM content of new systems and identify those that have complicated kinematics.

\section{DARK MATTER CONSTRAINTS}

We use the spectroscopically determined $J$-factors (when possible) and predicted J-factors (otherwise) for each confirmed and candidate dSph to interpret the $\gamma$-ray flux upper limits within a DM framework. Figure 6 summarizes the observed flux and $\langle\sigma v\rangle$ upper limits derived for individual confirmed and candidate dSphs, assuming a DM particle with a mass of $100 \mathrm{GeV}$ annihilating through the $b \bar{b}$-channel. ${ }^{48} \mathrm{We}$ find that the observed upper limits are consistent with expectations from blank-sky regions. We also show the median expected upper limit assuming that DM annihilates with a cross section comparable to the thermal relic cross section. Targets with $\log _{10}\left(\mathrm{~J} / \mathrm{GeV}^{2} \mathrm{~cm}^{-5}\right) \lesssim 18.3$ would have a negligible $\gamma$-ray signal for a DM cross section similar to the thermal relic value. However, the upper limits for systems with larger $J$-factors would be expected to deviate from the null hypothesis.

Given the large $J$-factors for Reticulum II (measured) and Tucana III (predicted) we consider whether the low-significance excess emission observed toward them is consistent with a DM annihilation signal (Table 2 and Figure 4). Several other confirmed and candidate dSphs have $J$-factors comparable to Reticulum II and Tucana III but have no excess over the background. The largest observed excess is associated with Indus II, which, at a distance of $214 \mathrm{kpc}$, has a predicted signal that is two orders of magnitude smaller than for the most promising candidates. In addition, evidence for tidal tails

\footnotetext{
${ }^{48}$ Results for both channels as well as bin-by-bin likelihood functions for each target are available in machine-readable format at: http://www-glast.stanford. edu/pub_data/1203/.
}

associated with Tucana III (Drlica-Wagner et al. 2015b) might indicate that the DM halo of this stellar system is being tidally stripped. Tidal stripping might significantly lower the $J$-factors of this target compared to the expectation when assuming hydrostatic equilibrium, which would decrease the predicted $\gamma$ ray flux from DM annihilation. Finally, based on an analysis of blank-sky regions and the number of targets considered, a maximum TS value at least as large as that observed is expected in the majority of background-only realizations (see column 7 of Table 2).

To further explore the consistency of the $\gamma$-ray data with a DM annihilation signal from the dSph population, and to increase search sensitivity, we combined observations of multiple satellite systems in a joint likelihood analysis. By simultaneously analyzing the population of confirmed and candidate dSphs, we avoid a look-elsewhere effect from focusing on excesses or deficits associated with individual targets. As opposed to weighting each target equally, the combined likelihood analysis emphasizes those targets with the largest $J$-factors and enforces consistency in the DM annihilation spectrum.

The current uncertainty in the photometric classification of newly found systems motivates the definition of three target samples for our combined analysis (Table 1).

1. Our "nominal" sample includes: (1) kinematically confirmed dSphs, and (2) systems with $r_{1 / 2}>20 \mathrm{pc}$ and $\mu>25$ mag $\operatorname{arcsec}^{-2}$.

2. We define a "conservative" sample as a sub-selection of the nominal sample excluding systems with kinematic or photometric indications of tidal disruption. Specifically, the conservative sample excludes Boötes III and Willman 1, which appear to be dSphs but have kinematics that are difficult to interpret (Carlin et al. 2009; Willman et al. 2011). Additionally, we exclude the new system Tucana III, which shows possible indication of tidal stripping (Drlica-Wagner et al. 2015b).

3. Finally, we define an "inclusive" sample, which augments the nominal sample selection with all systems with $r_{1 / 2}>10 \mathrm{pc}$ and $\mu>25 \mathrm{mag} \operatorname{arcsec}^{-2}$. This sample includes four ambiguous systems: Cetus II, Eridanus III, Kim 2, and Tucana V. 

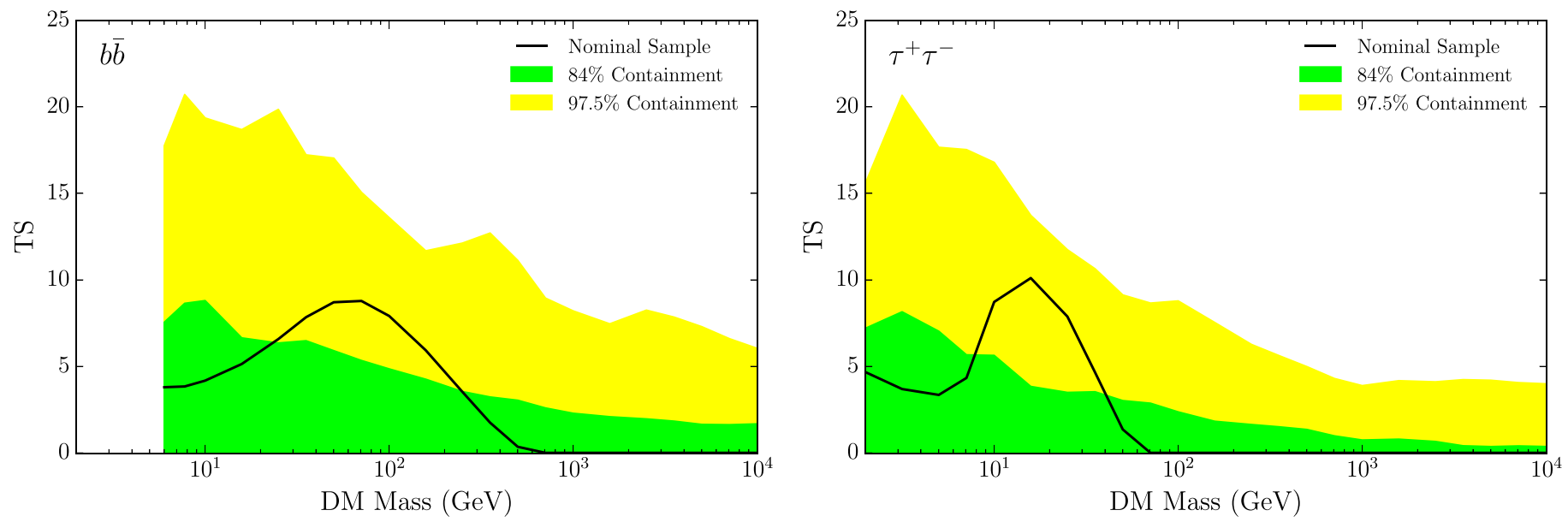

Figure 7. Local detection significance, expressed as a log-likelihood test statistic (TS), from the combined analysis of the nominal target sample assuming DM annihilation through the $b \bar{b}$ (left) or $\tau^{+} \tau^{-}$(right) channels. The log-normal $J$-factor uncertainties for targets lacking spectroscopic $J$-factors are 0.6 dex in this example. The bands represent the local one-sided $84 \%$ (green) and $97.5 \%$ (yellow) containment regions derived from 300 random sets of 45 blank-sky locations using the same set of $J$-factors as in the nominal sample.

These sample selections are compared to the photometric characteristics of dSphs and globular clusters in Figure 1 and are indicated in Table 1.

When analyzing the $\gamma$-ray data in the context of DM annihilation, we made use of measured $J$-factors based on spectroscopic observations when possible. If spectroscopic $J$-factors were unavailable, we used the values predicted from the distance scaling relationship and adopted a nominal uncertainty of 0.6 dex. We followed the prescription of Ackermann et al. (2015b) to incorporate the $J$-factor uncertainty as a nuisance parameter (see Equations (3)-(5) in Ackermann et al. 2015b). The largest excess found in the combined analysis of our nominal sample was TS $=10.1$ for a DM particle mass of $15.8 \mathrm{GeV}$ annihilating into $\tau$-leptons (see Figure 7). We calibrated this TS against a sample of randomly selected blank-sky locations to get $p_{\text {local }}=0.047(1.7 \sigma)$. We converted this to $p_{\text {global }}=0.23(0.7 \sigma)$ by applying a trials factor to account for our scan in DM mass and annihilation channel. $^{49}$

Ackermann et al. (2014) found that cross section upper limits derived from dSphs are fairly insensitive to the assumed spatial extension. However, we investigate the impact of modeling the targets as spatially extended sources using the Navarro-FrenkWhite (NFW) DM density profiles projected along the 1.o.s. (Navarro et al. 1997). Since the scale radii of the dSph candidates are not well constrained, we consider characteristic scale radii of $100 \mathrm{pc}, 316 \mathrm{pc}$, and $1 \mathrm{kpc}$. When assuming the largest scale radius of $1 \mathrm{kpc}$, we find that the TS of the most significant excess observed in the analysis of the nominal sample $\left(\tau^{+} \tau^{-}\right.$channel and mass of $\left.15.8 \mathrm{GeV}\right)$ increases to $\mathrm{TS}=15.3$. The global significance of the excess assuming the most extended spatial model is $p_{\text {global }}=0.21(0.8 \sigma)$; however, this value does not account for the additional trials factor from testing multiple spatial models.

We also performed our analysis using predicted $J$-factor uncertainties of 0.4 and 0.8 dex when spectroscopic $J$-factors were unavailable. The TS values and associated detection significances from these analyses are listed in Table 3 .

\footnotetext{
49 If we only tested the single DM model best-fit to the GCE then it would not be necessary to include a trials factor for testing multiple DM masses and channels (e.g., Hooper \& Linden 2015).
}

Table 3

Combined Analysis Results

\begin{tabular}{lcccc}
\hline \hline Sample & Channel & Mass $(\mathrm{GeV})$ & TS & $p_{\text {global }}$ \\
\hline \multicolumn{5}{c}{$0.4 \mathrm{dex}$} \\
\hline Inclusive & $\tau^{+} \tau^{-}$ & 15.8 & 8.5 & $0.20(0.8 \sigma)$ \\
Nominal & $\tau^{+} \tau^{-}$ & 15.8 & 8.5 & $0.18(0.9 \sigma)$ \\
Conservative & $\tau^{+} \tau^{-}$ & 15.8 & 2.5 & $0.51(-0.0 \sigma)$ \\
\hline \multicolumn{5}{c}{$0.6 \mathrm{dex}$} \\
\hline Inclusive & $\tau^{+} \tau^{-}$ & 15.8 & 10.1 & $0.27(0.6 \sigma)$ \\
Nominal & $\tau^{+} \tau^{-}$ & 15.8 & 10.1 & $0.23(0.7 \sigma)$ \\
Conservative & $\tau^{+} \tau^{-}$ & 15.8 & 3.0 & $0.60(-0.3 \sigma)$ \\
\hline & & $0.8 \mathrm{dex}$ & & \\
\hline Inclusive & $\tau^{+} \tau^{-}$ & 15.8 & 11.6 & $0.34(0.4 \sigma)$ \\
Nominal & $\tau^{+} \tau^{-}$ & 15.8 & 11.4 & $0.29(0.6 \sigma)$ \\
Conservative & $\tau^{+} \tau^{-}$ & 25.0 & 3.8 & $0.68(-0.5 \sigma)$ \\
\hline
\end{tabular}

Note. Largest TS values from the combined analysis of satellite systems in our three target samples. We adopt log-normal $J$-factor uncertainties of 0.4 dex, 0.6 dex, and $0.8 \mathrm{dex}$ for targets lacking spectroscopic $J$-factors. The global $p$-value is calibrated from random blank-sky regions and is corrected for a trials factor from fitting multiple DM annihilation spectra.

Different choices for the target sample and predicted $J$-factor uncertainties yield distinct null distributions for the TS. The resulting $p_{\text {global }}$ values do not account for the extra trials factor from testing multiple target samples and $J$-factor uncertainties. In all cases, $p_{\text {global }}<1 \sigma$. Due to the lack of a significant excess in the combined analysis, we conclude that there is no significant evidence of DM annihilation in the population of confirmed and candidate dSphs.

Assuming that the $J$-factors are an accurate representation of the expected DM annihilation signal, a combined analysis of the satellite population is more sensitive than the analysis of any individual target. In Figure 8, we show the median expected sensitivity for an analysis of our nominal sample assuming several different $J$-factor uncertainties for targets without spectroscopically determined $J$-factors (kinematic $J$-factors are held fixed in each case). Additionally, we show the optimistic scenario where the $J$-factors for the entire sample 

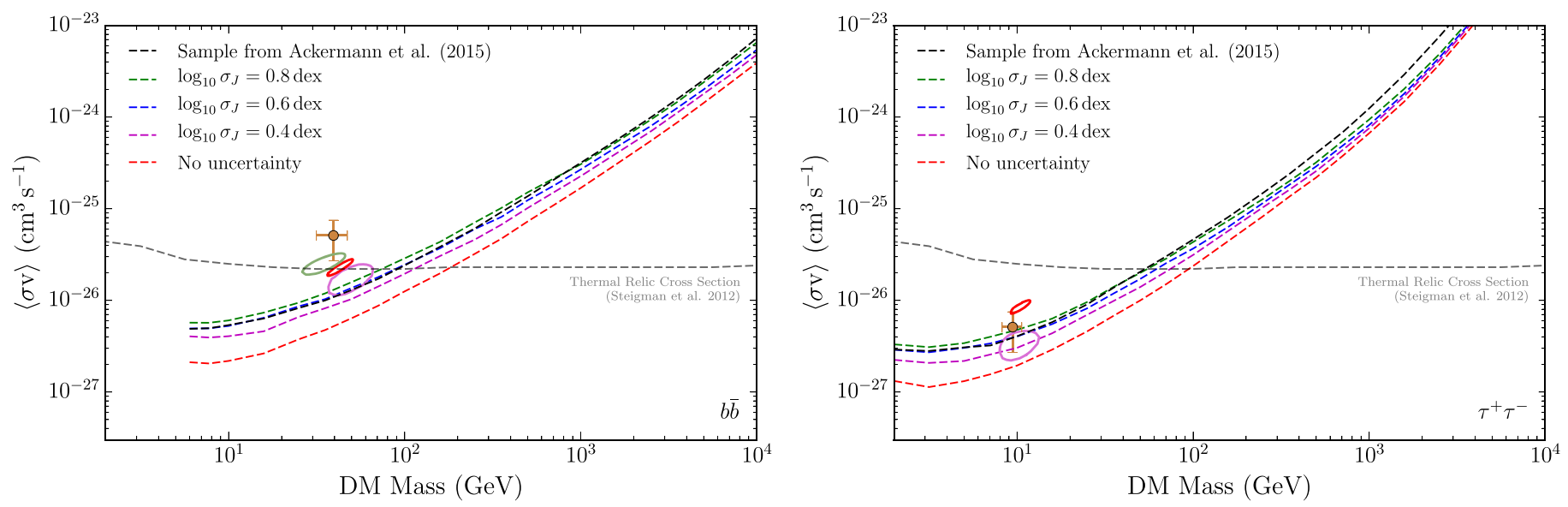

Figure 8. Expected sensitivity expressed as a limit on the DM annihilation cross section for the $b \bar{b}$ (left) and $\tau^{+} \tau^{-}$(right) channels. The expected sensitivity is calculated as the median 95\% confidence level upper limit from 300 sets of random blank-sky locations. The dashed black line shows the median expected sensitivity for the sample of $15 \mathrm{dSphs}$ with kinematic $J$-factors used in the combined analysis of Ackermann et al. (2015b). Colored dashed curves show the median sensitivity for the combined analysis of the nominal sample derived assuming $J$-factor uncertainties of 0.8 dex, 0.6 dex, and 0.4 dex for the targets with distance-based $J$-factor estimates. The "No Uncertainty" expectation curve is derived assuming zero $J$-factor uncertainty for all targets and represents the limiting sensitivity attainable by reducing $J$-factor uncertainties. The closed contours and marker show the best-fit regions (at $2 \sigma$ confidence) in cross-section and mass from several DM interpretations of the GCE: green contour (Gordon \& Macias 2013), red contour (Daylan et al. 2016), orange data point (Abazajian et al. 2014), purple contour (Calore et al. 2015). The dashed gray curve corresponds to the thermal relic cross section from Steigman et al. (2012).

can be determined exactly. In this limiting case, the analysis is sensitive to the thermal relic cross section for DM particles with mass $\lesssim 200 \mathrm{GeV}$, a factor of $\sim 2$ increase in mass relative to the analysis of Ackermann et al. (2015b).

In Figure 9 we show upper limits derived from a combined analysis of our nominal sample assuming a $J$-factor uncertainty of 0.6 dex for targets lacking spectroscopic $J$-factors. We find that the derived upper limits are consistent within the range of statistical fluctuation expected from 300 random high-latitude blank-sky fields. The derived upper limits lie above the median expectation for masses below $\sim 1 \mathrm{TeV}$ and $\sim 70 \mathrm{GeV}$ for the $b \bar{b}$ and $\tau^{+} \tau^{-}$channels, respectively. This behavior can be attributed to the low-significance excesses discussed in Section 3. In contrast, we note that the limits lie below the median expectation at higher masses.

\section{CONCLUSIONS}

We have performed a comprehensive $\gamma$-ray analysis of Fermi-LAT data coincident with 45 confirmed and candidate dSphs. We find no statistically significant $(>3 \sigma) \gamma$-ray excesses toward any of our targets. Four of the targets (including two nearby systems) exhibit small excesses with local significances $<2.5 \sigma$. Since the characteristics of the DM particle (i.e., mass and annihilation channel) are expected to be the same in all $\mathrm{dSphs}$, we perform a combined analysis on the sample of confirmed and candidate dSphs. We use a simple scaling relationship to predict the DM annihilation signal in systems without spectroscopic data. When considering the ensemble of targets, the $\gamma$-ray data are consistent with the background-only null hypothesis. The maximum excess found in a joint likelihood analysis of our nominal target sample yields a maximum global significance of $p_{\text {global }}=0.23(0.7 \sigma)$ for a DM mass of $15.8 \mathrm{GeV}$ annihilating via the $\tau^{+} \tau^{-}$channel.

We calculate the median expected sensitivity assuming the DM contents of the new candidate $\mathrm{dSphs}$ are comparable to those of previously known dSphs. The expected sensitivity to DM annihilation improves as more targets are added, and depends on the precision with which the $J$-factors of the new systems can be measured, as well as the DM mass and annihilation channel being tested. Assuming that the $J$-factors of the new systems can be measured with an uncertainty of 0.6 dex, the improvement in sensitivity is a factor of $\sim 1.5$ for hard annihilation spectra (e.g., the $\tau^{+} \tau^{-}$channel) compared to the median expected limits in Ackermann et al. (2015b). More precisely determined $J$-factors are expected to improve the sensitivity by up to a factor of 2 , motivating deeper spectroscopic observations both with current facilities and future $30 \mathrm{~m}$ class telescopes (Bernstein et al. 2014; Skidmore et al. 2015).

The limits derived from LAT data coincident with confirmed and candidate dSphs do not yet conclusively confirm or refute a DM interpretation of the GCE (Gordon \& Macias 2013; Abazajian et al. 2014; Calore et al. 2015; Daylan et al. 2016). Relative to the combined analysis of Ackermann et al. (2015b), the limits derived here are up to a factor of 2 more constraining at large DM masses $\left(m_{\mathrm{DM}, b \bar{b}} \gtrsim 1 \mathrm{TeV}\right.$ and $\left.m_{\mathrm{DM}, \tau^{+} \tau^{-}} \gtrsim 70 \mathrm{GeV}\right)$ and a factor of $\sim 1.5$ less constraining for lower DM masses. The weaker limits obtained at low DM mass can be attributed to low-significance excesses coincident with some of the nearby and recently discovered stellar systems, i.e., Reticulum II and Tucana III. While the excesses associated with these targets are broadly consistent with the DM spectrum and cross section fit to the GCE, we refrain from a more extensive DM interpretation due to the low significance of these excesses, the uncertainties in the $J$-factors of these targets, and the lack of any significant signal in the combined analysis.

Ongoing Fermi-LAT observations, more precise $J$-factor determinations with deeper spectroscopy, and searches for new dSphs in large optical surveys will each contribute to the future sensitivity of DM searches using Milky Way satellites (Charles et al. 2016). In particular, the Large Synoptic Survey Telescope (Ivezic et al. 2008) is expected to find hundreds of new Milky Way satellite galaxies (Tollerud et al. 2008; Hargis et al. 2014). Due to the difficulty in acquiring spectroscopic observations and the relative accessibility of $\gamma$-ray observations, it seems likely that $\gamma$-ray analysis will precede $J$-factor determinations in many cases. To facilitate updates to the DM search as spectroscopic $J$-factors become available, the likelihood 

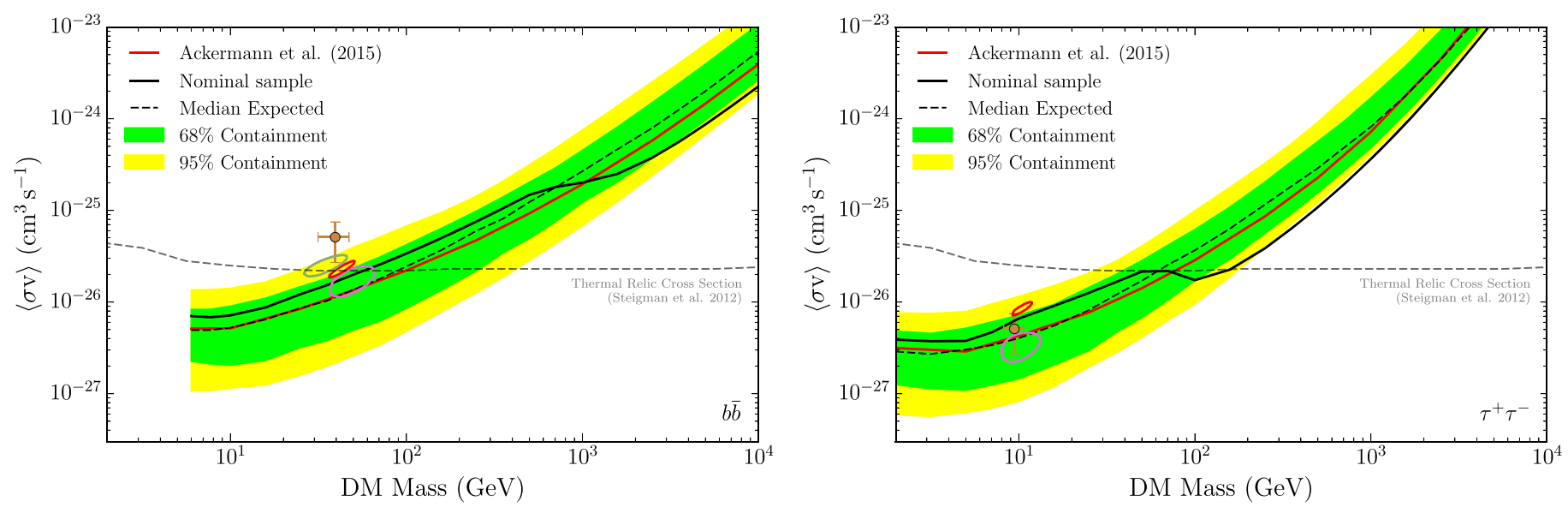

Figure 9. Upper limits (95\% confidence level) on the DM annihilation cross section derived from a combined analysis of the nominal target sample for the $b \bar{b}$ (left) and $\tau^{+} \tau^{-}$(right) channels. Bands for the expected sensitivity are calculated by repeating the same analysis on 300 randomly selected sets of high-Galactic-latitude blank fields in the LAT data. The dashed line shows the median expected sensitivity while the bands represent the 68\% and $95 \%$ quantiles. Spectroscopically measured $J$-factors are used when available; otherwise, $J$-factors are predicted photometrically with an uncertainty of 0.6 dex (solid red line). The solid red line shows the observed limit from the combined analysis of $15 \mathrm{dSphs}$ from Ackermann et al. (2015b). The closed contours and marker show the best-fit regions (at $2 \sigma$ confidence) in cross-section and mass from several DM interpretations of the GCE: green contour (Gordon \& Macias 2013), red contour (Daylan et al. 2016), orange data point (Abazajian et al. 2014), purple contour (Calore et al. 2015). The dashed gray curve corresponds to the thermal relic cross section from Steigman et al. (2012).
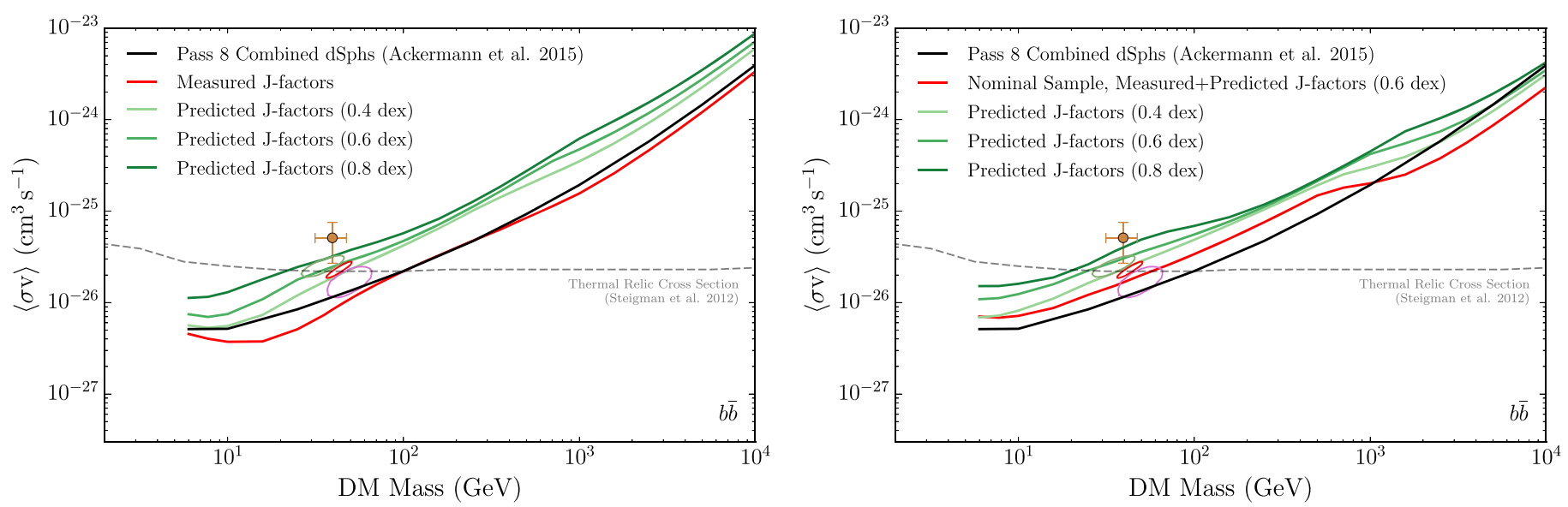

Figure 10. Upper limits on the DM annihilation cross section ( $b \bar{b}$ channel) derived from the sub-sample of dSphs with measured $J$-factors (left) and the complete nominal sample (right). Green curves show the limits obtained when these samples are analyzed using only predicted $J$-factors (even when measured $J$-factors are available) and fixed $J$-factor uncertainties of $0.4,0.6$, and 0.8 dex. The solid black line shows the observed limit from Ackermann et al. (2015b). The closed contours and marker are the same as depicted in Figures 8 and 9.

profiles for each energy bin used to derive our $\gamma$-ray flux upper limits will be made publicly available. We plan to augment this resource as more new systems are discovered.

After the completion of this analysis, we became aware of an independent study of LAT Pass 8 data coincident with DES Y2 dSph candidates ( $\mathrm{Li}$ et al. 2016). The $\gamma$-ray results associated with individual targets are consistent between the two works; however, the samples selected for combined analysis are different.

We would like to thank Tim Linden and Dan Hooper for helpful and engaging conversations. We also thank the anonymous referee for thoughtful and constructive feedback on this manuscript.

The Fermi LAT Collaboration acknowledges generous ongoing support from a number of agencies and institutes that have supported both the development and the operation of the LAT as well as scientific data analysis. These include the
National Aeronautics and Space Administration and the Department of Energy in the United States, the Commissariat à l'Energie Atomique and the Centre National de la Recherche Scientifique/Institut National de Physique Nucléaire et de Physique des Particules in France, the Agenzia Spaziale Italiana and the Istituto Nazionale di Fisica Nucleare in Italy, the Ministry of Education, Culture, Sports, Science and Technology (MEXT), High Energy Accelerator Research Organization (KEK) and Japan Aerospace Exploration Agency (JAXA) in Japan, and the K. A. Wallenberg Foundation, the Swedish Research Council and the Swedish National Space Board in Sweden. Additional support for science analysis during the operations phase is gratefully acknowledged from the Istituto Nazionale di Astrofisica in Italy and the Centre National d'Études Spatiales in France.

Funding for the DES Projects has been provided by the U.S. Department of Energy, the U.S. National Science Foundation, the Ministry of Science and Education of Spain, the Science 

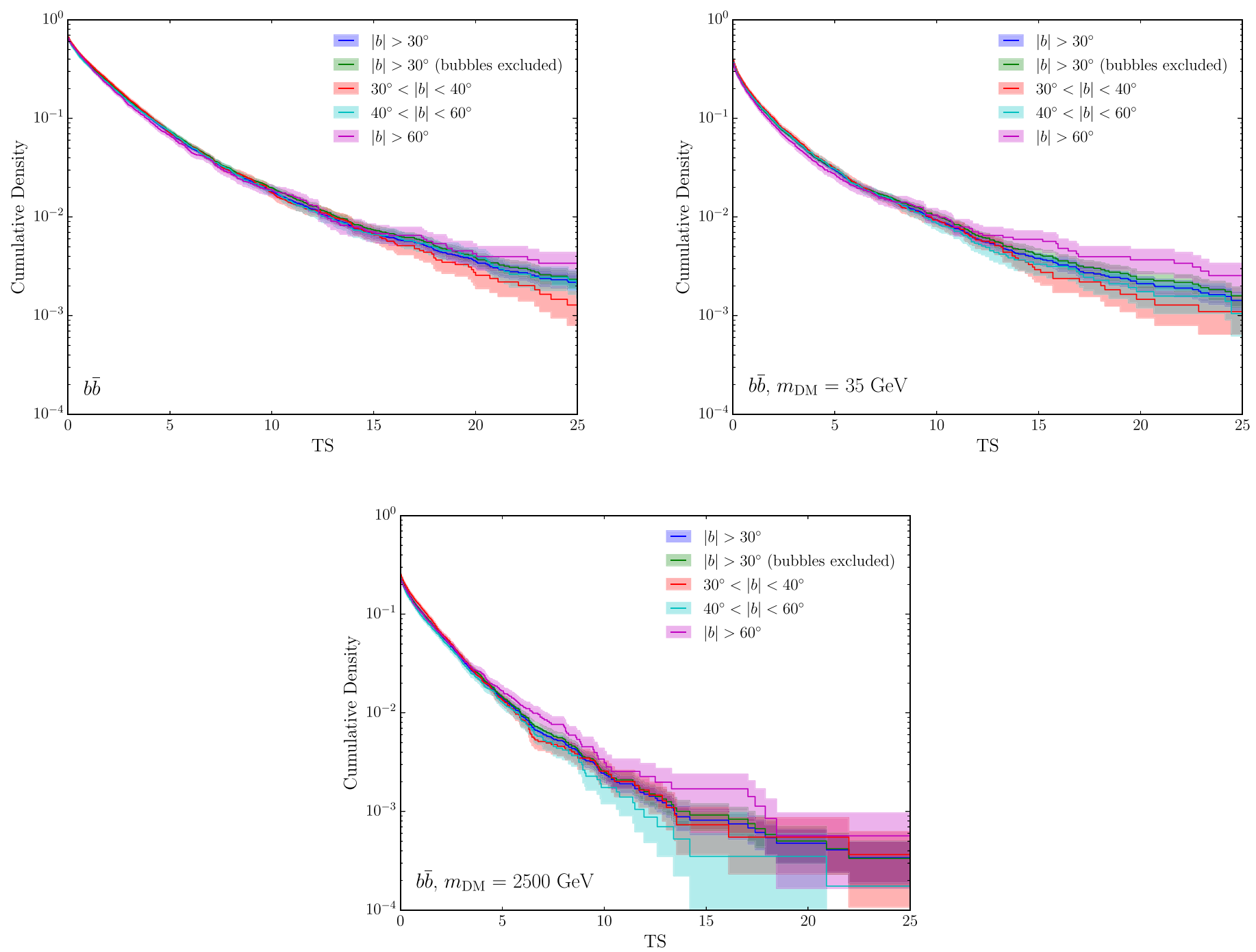

Figure 11. Cumulative TS distributions derived from blank-sky regions when fit with DM annihilation spectra (b $\bar{b}$ channel). Each curve corresponds to the distribution of blank-sky positions drawn from different subselections of the $|b|>30^{\circ}$ region. The bubbles excluded selection excludes the region containing the Fermi bubbles with $|\ell|<30^{\circ}$ and $|b|<60^{\circ}$. Shaded bands indicate the $1 \sigma$ uncertainties on the cumulative fraction. Top left: TS distribution for the best-fit (maximum TS) mass for each blank-sky position and realization. Top right: TS distribution for a DM mass of $35.4 \mathrm{GeV}$. Bottom: TS distribution for a DM mass of 2.5 TeV.

and Technology Facilities Council of the United Kingdom, the Higher Education Funding Council for England, the National Center for Supercomputing Applications at the University of Illinois at Urbana-Champaign, the Kavli Institute of Cosmological Physics at the University of Chicago, the Center for Cosmology and Astro-Particle Physics at the Ohio State University, the Mitchell Institute for Fundamental Physics and Astronomy at Texas A\&M University, Financiadora de Estudos e Projetos, Fundação Carlos Chagas Filho de Amparo à Pesquisa do Estado do Rio de Janeiro, Conselho Nacional de Desenvolvimento Científico e Tecnológico and the Ministério da Ciência, Tecnologia e Inovação, the Deutsche Forschungsgemeinschaft and the Collaborating Institutions in the DES. The DES data management system is supported by the National Science Foundation under Grant Number AST-1138766. The DES participants from Spanish institutions are partially supported by MINECO under grants AYA2012-39559, ESP2013-48274, FPA2013-47986, and Centro de Excelencia Severo Ochoa SEV-2012-0234, some of which include ERDF funds from the European Union.
The Collaborating Institutions are Argonne National Laboratory, the University of California at Santa Cruz, the University of Cambridge, Centro de Investigaciones Enérgeticas, Medioambientales y Tecnológicas-Madrid, the University of Chicago, University College London, the DES-Brazil Consortium, the University of Edinburgh, the Eidgenössische Technische Hochschule (ETH) Zürich, Fermi National Accelerator Laboratory, the University of Illinois at Urbana-Champaign, the Institut de Ciències de l'Espai (IEEC/CSIC), the Institut de Física d'Altes Energies, Lawrence Berkeley National Laboratory, the Ludwig-Maximilians Universität München and the associated Excellence Cluster universe, the University of Michigan, the National Optical Astronomy Observatory, the University of Nottingham, The Ohio State University, the University of Pennsylvania, the University of Portsmouth, SLAC National Accelerator Laboratory, Stanford University, the University of Sussex, and Texas A\&M University.

This research has made use of the NASA/IPAC Extragalactic Database (NED) which is operated by the Jet Propulsion Laboratory, California Institute of Technology, under contract with the National Aeronautics and Space Administration.

Facilities: Fermi, Blanco. 


\section{APPENDIX}

\section{A.1. Influence of Predicted J-factors}

To characterize the influence on the observed limits due to targets lacking measured $J$-factors, we perform an a posteriori examination of different subsets of the target sample. Analyzing only the dSphs with measured $J$-factors yields results that are similar to those of Ackermann et al. (2015b) because the target sample is nearly identical (Figure 10). The primary differences between these two analyses are the method for calculating $J$-factors and their uncertainties (Martinez 2015 versus GeringerSameth et al. 2015b), the spatial model of the dSphs (spatially extended NFW versus point-like), and the addition of the dSph Reticulum II. As shown in the left panel of Figure 10, the limits derived with the subset of dSphs in the nominal sample that have measured $J$-factors deviate by at most a factor of two from the limits of Ackermann et al. (2015b).

Replacing the measured $J$-factors of all dSphs with the predicted $J$-factors from Table 1 has a somewhat larger effect on the observed limits than using only $\mathrm{dSphs}$ with measured $J$-factors. The green curves in Figure 10 show the composite limits evaluated using only predicted $J$-factors for two different target samples: the subset of the nominal sample with measured $J$-factors (left panel) and the full nominal sample (right panel). Using only predicted $J$-factors weakens the observed limits by a factor of 2-3 depending on the choice of $J$-factor uncertainty.

The weakening of the limits when using predicted $J$-factors can be partially attributed to statistical fluctuations that occur when changing the relative weighting of objects in the sample. As a population, the predicted $J$-factors are statistically consistent with the measured $J$-factors of Geringer-Sameth et al. (2015b, see Figure 5). However, on an object-by-object basis, the differences in $J$-factors can substantially change the weight given to positive or negative residuals observed at the location of a given $\mathrm{dSph}$. We also note that the limits evaluated with predicted $J$-factors are consistent with the $95 \%$ expectation band from Figure 9 (i.e., the expected variation from statistical fluctuations).

\section{A.2. Effect of Variations in Diffuse Background Intensity}

To assess the potential impact of variations in the diffuse background intensity on the derived TS distribution, we have generated TS distributions from different subselections of the blank-sky regions. As shown in Figure 11, there is no statistically significant change in the TS distribution when excluding regions around the Fermi bubbles or selecting random sky positions from lower or higher Galactic latitudes. We conclude that the trial factor calculation is robust to local variations in the background intensity at the level found in the high-latitude sky.

\section{REFERENCES}

Abazajian, K. N., Canac, N., Horiuchi, S., \& Kaplinghat, M. 2014, PhRvD, 90, 023526

Abdo, A. A., Ackermann, M., Ajello, M., et al. 2010, ApJ, 712, 147

Acero, F., Ackermann, M., Ajello, M., et al. 2015, ApJS, 218, 23

Ackermann, M., Ajello, M., Albert, A., et al. 2011, PhRvL, 107, 241302

Ackermann, M., Ajello, M., Atwood, W. B., et al. 2015a, ApJ, 810, 14

Ackermann, M., Albert, A., Anderson, B., et al. 2014, PhRvD, 89, 042001
Ackermann, M., Albert, A., Anderson, B., et al. 2015b, PhRvL, 115, 231301

Ajello, M., Albert, A., Atwood, W. B., et al. 2016, ApJ, 819, 44

Atwood, W. B., Abdo, A. A., Ackermann, M., et al. 2009, ApJ, 697, 1071

Baltz, E., Berenji, B., Bertone, G., et al. 2008, JCAP, 0807, 013

Bechtol, K., Drlica-Wagner, A., Balbinot, E., et al. 2015, ApJ, 807, 50

Bernstein, R. A., McCarthy, P. J., Raybould, K., et al. 2014, Proc. SPIE, 9145, 91451C

Bertone, G., Hooper, D., \& Silk, J. 2005, PhR, 405, 279

Bonnivard, V., Combet, C., Daniel, M., et al. 2015a, MNRAS, 453, 849

Bonnivard, V., Combet, C., Maurin, D., et al. 2015b, ApJL, 808, L36

Bradford, J. D., Geha, M., Muoz, R., et al. 2011, ApJ, 743, 167 (Erratum: 2013, ApJ, 778, 85)

Calore, F., Cholis, I., \& Weniger, C. 2015, JCAP, 1503, 038

Carlin, J. L., Grillmair, C. J., Munoz, R. R., Nidever, D. L., \& Majewski, S. R. 2009, ApJL, 702, L9

Carlson, E., Hooper, D., \& Linden, T. 2015, PhRvD, 91, 061302

Charles, E., Sánchez-Conde, M., Anderson, B., et al. 2016, PhR, 636, 1

D’Abrusco, R., Massaro, F., Paggi, A., et al. 2014, ApJS, 215, 14

Daylan, T., Finkbeiner, D. P., Hooper, D., et al. 2016, PDU, 12, 1

DES Collaboration 2005, arXiv:astro-ph/0510346

Drlica-Wagner, A., Albert, A., Bechtol, K., et al. 2015a, ApJL, 809, L4

Drlica-Wagner, A., Bechtol, K., Rykoff, E. S., et al. 2015b, ApJ, 813, 109

Evans, N. W., Ferrer, F., \& Sarkar, S. 2004, PhRvD, 69, 123501

Fadely, R., Willman, B., Geha, M., et al. 2011, AJ, 142, 88

Feng, J. L. 2010, ARA\&A, 48, 495

Flaugher, B., Diehl, H. T., Honscheid, K., et al. 2015, AJ, 150, 150

Frinchaboy, P. M., Majewski, S. R., Muñoz, R. R., et al. 2012, ApJ, 756, 74

Geha, M., Willman, B., Simon, J. D., et al. 2009, ApJ, 692, 1464

Geringer-Sameth, A., \& Koushiappas, S. M. 2011, PhRvL, 107, 241303

Geringer-Sameth, A., Koushiappas, S. M., \& Walker, M. 2015a, ApJ, 801,74

Geringer-Sameth, A., Koushiappas, S. M., \& Walker, M. G. 2015b, PhRvD, 91, 083535

Geringer-Sameth, A., Walker, M. G., Koushiappas, S. M., et al. 2015c, PhRvL, 115,081101

Gondolo, P., Edsjo, J., Ullio, P., et al. 2004, JCAP, 0407, 008

Gordon, C., \& Macias, O. 2013, PhRvD, 88, 083521

Hargis, J. R., Willman, B., \& Peter, A. H. G. 2014, ApJL, 795, L13

Harris, W. E. 1996, AJ, 112, 1487

Healey, S. E., Romani, R. W., Taylor, G. B., et al. 2007, ApJS, 171, 61

Healey, S. E., Romani, R. W., Cotter, G., et al. 2008, ApJS, 175, 97

Hooper, D., \& Linden, T. 2015, JCAP, 1509, 016

Ivezic, Z., Tyson, J., Allsman, R., Andrew, J., \& Angel, R. 2008, arXiv:0805.2366

Jeltema, T. E., \& Profumo, S. 2008, JCAP, 0811, 003

Jordi, K., Grebel, E. K., Hilker, M., et al. 2009, AJ, 137, 4586

Kim, D., \& Jerjen, H. 2015, ApJ, 808, L39

Kim, D., Jerjen, H., Mackey, D., Da Costa, G. S., \& Milone, A. P. 2015a, ApJ, 804, L44

Kim, D., Jerjen, H., Milone, A. P., Mackey, D., \& Da Costa, G. S. 2015b, ApJ, 803,63

Kirby, E. N., Boylan-Kolchin, M., Cohen, J. G., et al. 2013, ApJ, 770, 16

Kirby, E. N., Cohen, J. G., Simon, J. D., \& Guhathakurta, P. 2015a, ApJ, 814, L7

Kirby, E. N., Simon, J. D., \& Cohen, J. G. 2015b, ApJ, 810, 56

Koposov, S. E., Belokurov, V., Torrealba, G., \& Evans, N. W. 2015a, ApJ, 805, 130

Koposov, S. E., Casey, A. R., Belokurov, V., et al. 2015b, ApJ, 811, 62

Laevens, B. P. M., Martin, N. F., Bernard, E. J., et al. 2015a, ApJ, 813,44

Laevens, B. P. M., Martin, N. F., Ibata, R. A., et al. 2015b, ApJL, 802, L18

Laevens, B. P. M., Martin, N. F., Sesar, B., et al. 2014, ApJL, 786, L3

Li, S., Liang, Y.-F., Duan, K.-K., et al. 2016, PhRvD, 93, 043518

Luque, E., Queiroz, A., Santiago, B., et al. 2016, MNRAS, 458, 603

Martin, N. F., Ibata, R. A., Bellazzini, M., et al. 2004, MNRAS, 348, 12

Martin, N. F., Geha, M., Ibata, R. A., et al. 2015a, MNRAS, 458, 59

Martin, N. F., Ibata, R. A., Collins, M. L. M., et al. 2016, ApJ, 818, 40

Martin, N. F., Nidever, D. L., Besla, G., et al. 2015b, ApJ, 804, L5

Martinez, G. D. 2015, MNRAS, 451, 2524

Massaro, E., Giommi, P., Leto, C., et al. 2009, A\&A, 495, 691

Massaro, F., D'Abrusco, R., Ajello, M., Grindlay, J. E., \& Smith, H. A. 2011, ApJL, 740, L48

Mazziotta, M. N., Loparco, F., de Palma, F., \& Giglietto, N. 2012, APh, 37, 26 
Navarro, J. F., Frenk, C. S., \& White, S. D. 1997, ApJ, 490, 493

Planck Collaboration 2015, A\&A, 594, A13

Simon, J. D., Drlica-Wagner, A., Li, T. S., et al. 2015, ApJ, 808, 95

Simon, J. D., \& Geha, M. 2007, ApJ, 670, 313

Simon, J. D., Geha, M., Minor, Q. E., et al. 2011, ApJ, 733, 46

Skidmore, W., et al. 2015, RAA, 15, 1945

Steigman, G., Dasgupta, B., \& Beacom, J. F. 2012, PhRvD, 86, 023506

Strigari, L. E., Bullock, J. S., Kaplinghat, M., et al. 2008, Nature, 454, 1096
Tollerud, E. J., Bullock, J. S., Strigari, L. E., \& Willman, B. 2008, ApJ, 688, 277

Walker, M. G., Mateo, M., \& Olszewski, E. W. 2009, AJ, 137, 3100

Walker, M. G., Mateo, M., Olszewski, E. W., et al. 2015a, ApJ, 808, 108

Walker, M. G., Mateo, M., Olszewski, E. W., et al. 2015b, ApJ, 819, 53

Willman, B., Geha, M., Strader, J., et al. 2011, AJ, 142, 128

Wright, A. E., Griffith, M. R., Burke, B. F., \& Ekers, R. D. 1994, ApJS, 91,111

York, D. G., Adelman, J., Anderson, J. E., et al. 2000, AJ, 120, 1579 